DRAFT VERSION NOVEMBER 23, 2018

Preprint typeset using LTEX style emulateapj v. 03/07/07

\title{
IDENTIFICATIONS AND PHOTOMETRIC REDSHIFTS OF THE 2 MS CHANDRA DEEP FIELD-SOUTH SOURCES
}

\author{
B. Luo,${ }^{1}$ W. N. Brandt, ${ }^{1}$ Y. Q. Xue,${ }^{1}$ M. Brusa,${ }^{2,3}$ D. M. Alexander,${ }^{4}$ F. E. Bauer,${ }^{5,6}$ A. Comastri, ${ }^{7}$ A. KoeKemoer,${ }^{8}$ \\ B. D. Lehmer, ${ }^{4,9,10}$ V. MAinieri, ${ }^{11}$ D. A. RafFerty, ${ }^{1}$ D. P. Schneider,${ }^{1}$ J. D. Silverman, ${ }^{12}$ \& C. VignAli ${ }^{13}$ \\ Draft version November 23, 2018
}

\begin{abstract}
We present reliable multiwavelength identifications and high-quality photometric redshifts for the $462 \mathrm{X}$-ray sources in the $\approx 2$ Ms Chandra Deep Field-South survey. Source identifications are carried out using deep optical-to-radio multiwavelength catalogs, and are then combined to create lists of primary and secondary counterparts for the X-ray sources. We identified reliable counterparts for $442(95.7 \%)$ of the X-ray sources, with an expected false-match probability of $\approx 6.2 \%$; we also selected four additional likely counterparts. The majority of the other $16 \mathrm{X}$-ray sources appear to be off-nuclear sources, sources associated with galaxy groups and clusters, high-redshift active galactic nuclei (AGNs), or spurious X-ray sources. A likelihood-ratio method is used for source matching, which effectively reduces the false-match probability at faint magnitudes compared to a simple error-circle matching method. We construct a master photometric catalog for the identified X-ray sources including up to 42 bands of UV-to-infrared data, and then calculate their photometric redshifts (photo-z's). High accuracy in the derived photo-z's is accomplished owing to (1) the up-to-date photometric data covering the full spectral energy distributions (SEDs) of the X-ray sources, (2) more accurate photometric data as a result of source deblending for $\approx 10 \%$ of the sources in the infrared bands and a few percent in the optical and near-infrared bands, (3) a set of 265 galaxy, AGN, and galaxy/AGN hybrid templates carefully constructed to best represent all possible SEDs, (4) the Zurich Extragalactic Bayesian Redshift Analyzer (ZEBRA) used to derive the photo-z's, which corrects the SED templates to best represent the SEDs of real sources at different redshifts and thus improves the photo-z quality. The reliability of the photo-z's is evaluated using the subsample of 220 sources with secure spectroscopic redshifts. We achieve an accuracy of $|\Delta z| /(1+z) \approx 1 \%$ and an outlier [with $|\Delta z| /(1+z)>0.15$ ] fraction of $\approx 1.4 \%$ for sources with spectroscopic redshifts. We performed blind tests to derive a more realistic estimate of the photo-z quality for sources without spectroscopic redshifts. We expect there are $\approx 9 \%$ outliers for the relatively brighter sources $(R \lesssim 26)$, and the outlier fraction will increase to $\approx 15-25 \%$ for the fainter sources $(R \gtrsim 26)$. The typical photo-z accuracy is $\approx 6-7 \%$. The outlier fraction and photo-z accuracy do not appear to have a redshift dependence (for $z \approx 0-4$ ). These photo-z's appear to be the best obtained so far for faint X-ray sources, and they have been significantly ( $\gtrsim 50 \%)$ improved compared to previous estimates of the photo-Z's for the X-ray sources in the $\approx 2$ Ms Chandra Deep Field-North and $\approx 1$ Ms Chandra Deep Field-South.
\end{abstract}

Subject headings: cosmology: observations — galaxies: active — galaxies: distances and redshifts — galaxies: photometry - X-rays: galaxies

\section{INTRODUCTION}

The Chandra Deep Field-North (CDF-N) and Chandra Deep Field-South (CDF-S) are the two deepest Chandra

\footnotetext{
${ }^{1}$ Department of Astronomy \& Astrophysics, 525 Davey Lab, The Pennsylvania State University, University Park, PA 16802, USA

${ }^{2}$ Max-Planck-Institut für Extraterrestrische Physik, Giessenbachstrasse, D-85748 Garching b. München, Germany

${ }^{3}$ University of Maryland, Baltimore County, 1000 Hilltop Circle, Baltimore, MD 21250, USA

${ }^{4}$ Department of Physics, Durham University, Durham, DH1 3LE, UK

5 Space Science Institute, 4750 Walnut Street, Suite 205, Boulder, Colorado 80301

${ }^{6}$ Pontificia Universidad Católica de Chile, Departamento de Astronomía y Astrofísica, Casilla 306, Santiago 22, Chile

${ }^{7}$ INAF-Osservatorio Astronomico di Bologna, Via Ranzani 1, Bologna, Italy

${ }^{8}$ Space Telescope Science Institute, 3700 San Martin Drive, Baltimore, MD 21218, USA

9 The Johns Hopkins University, Homewood Campus, Baltimore, MD 21218, USA

${ }^{10}$ NASA Goddard Space Flight Centre, Code 662, Greenbelt, MD 20771, USA

${ }^{11}$ European Southern Observatory, Karl-Schwarzschild-Strasse 2, Garching, D-85748, Germany

${ }^{12}$ Institute for the Physics and Mathematics of the Universe (IPMU), University of Tokyo, Kashiwanoha 5-1-5, Kashiwa, Chiba 277-8568, Japan

${ }^{13}$ Universitá di Bologna, Via Ranzani 1, Bologna, Italy
}

surveys (see Brandt \& Hasinger 2005 for a review), each covering $\approx 440 \mathrm{arcmin}^{2}$ areas with enormous multiwavelength observational investments. Together they have detected about 1160 X-ray point sources (e.g., Giacconi et al. 2002; Alexander et al. 2003; Luo et al. 2008, hereafter L08), with a sky density of $\approx 10000 \mathrm{deg}^{-2}$ at the limiting flux of

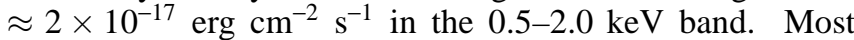
$(\approx 75 \%)$ of these $\mathrm{X}$-ray sources are active galactic nuclei (AGNs), often obscured, at $z \approx 0.1-4$; at faint fluxes, some of the sources are starburst and normal galaxies at $z \approx 0.1-2$ (e.g., Bauer et al. 2004).

The CDF-N and CDF-S provide an ideal opportunity to study AGN cosmological evolution, AGN physics, the role of AGNs in galaxy evolution, and the X-ray properties of starburst and normal galaxies, groups and clusters of galaxies, large-scale structures in the distant universe, and Galactic stars. However, one prerequisite of such research is to associate the X-ray sources with their correct counterparts at optical, near-infrared (NIR), infrared (IR), and radio wavelengths, and then to determine their redshifts either spectroscopically or photometrically. The CDF-S was recently expanded from $\approx 1 \mathrm{Ms}$ to $\approx 2 \mathrm{Ms}$ exposure, and more than 130 new X-ray sources are detected (L08). Meanwhile, additional deep multiwavelength surveys of the CDF-S have become 
available, including the VLA radio (e.g., Kellermann et al. 2008; Miller et al. 2008), Spitzer IR (e.g., Damen et al. 2010), NIR $K$-band (e.g., Grazian et al. 2006; Taylor et al. 2009), and GALEX UV (e.g. Morrissey et al.2005) surveys; there are also a few recent spectroscopic surveys that provide updated redshift information (e.g., Vanzella et al. 2008; Popesso et al. 2009). It is thus crucial to provide up-to-date X-ray source identifications in the CDF-S along with their redshifts to facilitate follow-up scientific studies.

One major uncertainty about X-ray source identifications in deep surveys is the contamination from spurious matches, e.g., because of the high source density in deep optical catalogs. For example, Barger et al. (2003) were able to assign optical counterparts to $\approx 85 \%$ of the $\mathrm{X}$-ray sources in the $\approx 2$ Ms CDF-N. However, they estimated the falsematch probability to be $\approx 10 \%$ at $R \lesssim 24$, and it will rise to $\approx 25 \%$ at $R \approx 24-26$. A simple error-circle method was used in their work, in which the closest optical source within a given matching radius of an X-ray source was selected as the counterpart. Recently, a more sophisticated likelihoodratio matching approach was adopted for matching X-ray sources in deep Chandra and XMM-Newton surveys to optical/NIR sources (e.g., Brusa et al. 2005; Cardamone et al. 2008; Laird et al. 2009; Aird et al. 2010). The likelihoodratio technique takes into account the positional errors of both the optical and X-ray sources and also the expected magnitude distribution of the counterparts, and thus it can significantly reduce the false-match probability at faint magnitudes. Furthermore, performing the source matching at some wavelengths other than optical, e.g., NIR or radio, can also reduce the false-match probability, as the X-ray sources may be better associated with NIR or radio sources. Combining the multiwavelength X-ray source identifications will effectively lower the overall false-match probability.

As many X-ray sources in deep surveys are optically faint (and are therefore challenging targets to obtain spectroscopic redshifts), a significant portion of the sources have their redshifts determined photometrically. Photometric redshifts are derived by fitting spectral energy distribution (SED) templates to the observed broadband photometric data, and their accuracy is largely controlled by the quality of the data available. The previous photometric redshifts for the X-ray sources in the $\approx 2$ Ms CDF-N (e.g., Barger et al. 2003; Capak et al. 2004) and $\approx 1$ Ms CDF-S (e.g., Wolf et al. 2004; Zheng et al. 2004 ) are generally accurate to $\approx 10 \%$ with $\approx 15-25 \%$ catastrophic redshift failures. The superb and improved multiwavelength coverage in the CDF-S has produced full SED sampling for the X-ray sources, from UV to IR, allowing determination of photometric redshifts to significantly higher accuracy.

In this paper, we present multiwavelength identifications of the CDF-S X-ray sources in the main source catalog of L08. Given the counterpart information, we compose a photometric catalog for these X-ray sources including up to 42 bands of UV-to-IR data. Photometric redshifts are then calculated and compared to the latest secure spectroscopic redshifts to evaluate their accuracy. The identifications and spectroscopic redshifts presented here supersede the counterpart and redshift information provided in L08. In $\S 2$ we describe in detail the likelihood-ratio matching method and our multiwavelength identification results. In $\S 3$ we present the photometric catalog and the derivation of photometric redshifts. The accuracy of the photometric redshifts is appropriately estimated. In $\$ 4$ we discuss the nature of the unidentified X-ray sources, and present some future prospects toward source identifications. We summarize in $\S 5$.

Throughout this paper, all magnitudes are based upon the AB magnitude system (e.g., Oke \& Gunn 1983), and we adopt the latest cosmology with $H_{0}=70.5 \mathrm{~km} \mathrm{~s}^{-1} \mathrm{Mpc}^{-1}, \Omega_{\mathrm{M}}=$ 0.274 , and $\Omega_{\Lambda}=0.726$ derived from the five-year WMAP observations (Komatsu et al. 2009).

\section{MULTIWAVELENGTH IDENTIFICATIONS OF THE 2 MS CDF-S $\mathrm{X}$-RAY SOURCES}

\subsection{X-ray and Optical-to-Radio Data}

The X-ray sample we chose is the main Chandra source catalog for the $\approx 2$ Ms CDF-S, which contains 462 X-ray sources (L08). These sources were detected in three X-ray bands, $0.5-8.0 \mathrm{keV}$ (full band), $0.5-2.0 \mathrm{keV}$ (soft band), and 2-8 keV (hard band), with a WAVDETECT false-positive probability threshold of $1 \times 10^{-6}$. The resulting source lists were then merged to create the main catalog (see $\$ 3.3$ of L08 for details). The survey covers an area of $\approx 436 \mathrm{arcmin}^{2}$ and reaches on-axis sensitivity limits of $\approx 7.1 \times 10^{-17}, \approx 1.9 \times 10^{-17}$ and $\approx 1.3 \times 10^{-16} \mathrm{erg} \mathrm{cm}^{-2} \mathrm{~s}^{-1}$ for the full, soft, and hard bands, respectively.

We have visually checked the positions of all the X-ray sources in L08 during an X-ray spectral extraction process (Bauer et al. 2010, in preparation), using the $\approx 2$ Ms CDFS Chandra events file and sometimes also the $\approx 250 \mathrm{ks}$ Extended Chandra Deep Field-South (E-CDF-S; Lehmer et al. 2005) events file for sources with large off-axis angles. Although most X-ray sources in L08 have good positions (no positional offsets or offsets $<0.24^{\prime \prime}$ ), there are five sources having positions off by $>1^{\prime \prime}$. These are sources $62,90,125$, 338 , and 456 , and we shifted their positions by $1.2^{\prime \prime}, 3.2^{\prime \prime}$, $1.3^{\prime \prime}, 2.8^{\prime \prime}$, and $1.5^{\prime \prime}$, respectively. Sources 90 and 456 are located at large off-axis angles $\left(11.8^{\prime}\right.$ and $\left.9.8^{\prime}\right)$; the other three sources are faint $(\approx 21-26$ detected counts in the soft band with errors of about 8-10 counts), and might be composite sources (blended with a weak nearby object). The complex point spread functions or morphologies of these five sources make it difficult to determine the centroid positions, and their positions reported in L08 are poorly defined. ${ }^{14}$

We searched for counterparts of the $\approx 2$ Ms CDF-S X-ray sources in several deep optical-to-radio catalogs, most of which cover the entire CDF-S region. Due to dust absorption and redshift effects, X-ray sources in deep surveys usually appear brighter at redder wavelengths. ${ }^{15}$ Therefore we used the following catalogs for identification purposes.

1. The WFI $R$-band catalog (Giacconi et al. 2002; Giavalisco et al. 2004), with a $5 \sigma$ limiting AB magnitude of 27.3 .

2. The GOODS-S HST version r2.0z z-band catalog (Giavalisco et al. 2004) ${ }^{16}$, with a $5 \sigma$ limiting AB magnitude of 28.2. The GOODS-S survey covers a solid angle of $\approx 160 \operatorname{arcmin}^{2}$ in the center of the CDF-S.

3. The GEMS HST z-band catalog (Caldwell et al. 2008), with a $5 \sigma$ limiting AB magnitude of 27.3. The GEMS

\footnotetext{
14 Four of these sources (all but source 456) do not have any apparent counterparts at their original positions.

15 Counterpart searching has also been performed at several shorter wavelengths (e.g., $V$ and $U$ bands), which yield fewer identifications.

${ }^{16}$ See http: //archive.stsci.edu/pub/hlsp/goods/catalog_r2/. The $2.0 \mathrm{z}$ catalog is based on the v2.0 GOODS-S images, which have significantly longer total exposure times in the $z$ band than the v1.0 images.
} 
survey was designed to exclude the GOODS-S region, and thus does not cover the entire CDF-S. However, Caldwell et al. (2008) reduced the GOODS-S v1.0 data identically to the reduction of GEMS, and the published $z$-band catalog covers the entire CDF-S. ${ }^{17}$

4. The GOODS-S MUSIC $K$-band catalog (Grazian et al. $2006)$, with a $90 \%(\approx 1.8 \sigma)$ limiting $\mathrm{AB}$ magnitude of 23.8. The GOODS-S MUSIC survey covers a solid angle of $\approx 140 \mathrm{arcmin}^{2}$ in the center of the CDF-S. The V2.0 catalog was used (A. Grazian 2009, private communication).

5. The MUSYC $K$-band catalog (Taylor et al. 2009), with a $5 \sigma$ limiting $\mathrm{AB}$ magnitude of 22.4. This catalog is not as deep as the GOODS-S MUSIC $K$-band catalog, but it covers the entire CDF-S.

6. The SIMPLE $3.6 \mu \mathrm{m}$ catalog (Damen et al. 2010), with a $5 \sigma$ limiting $\mathrm{AB}$ magnitude of 23.8. ${ }^{18}$

7. The VLA $1.4 \mathrm{GHz}$ radio catalog from Miller et al. (2008). We used the $5 \sigma$ catalog provided by N.A. Miller (2009, private communication), with a $5 \sigma \mathrm{lim}$ iting flux density of $\approx 40 \mu \mathrm{Jy}$. Compared to the optical positions (WFI $R$ band or GOODS-S $z$ band), the radio positions have small systematic offsets in right ascension and declination; since the X-ray positions were registered to the optical positions (L08), we manually shifted all the radio positions by $-0.18^{\prime \prime}$ in right ascension and $0.28^{\prime \prime}$ in declination to remove the systematic offsets.

The basic optical/NIR/IR/radio (hereafter ONIR) catalog information is listed in Table 1 Note that the GOODS-S and MUSIC catalogs do not cover the entire CDF-S. We also include in Table 1 the number of sources in each of these catalogs that are within the $\approx 2 \mathrm{Ms}$ CDF-S region $\left(N_{\mathrm{o}}\right)$. The locations of the optical, NIR, and IR bands in a sample SED are shown in Figure 1

\subsection{Matching Method}

Source matching to the X-ray catalog was performed for each of the optical-to-radio catalogs described above. We used the likelihood-ratio technique to identify the ONIR counterparts of the X-ray sources (e.g., Sutherland \& Saunders 1992; Ciliegi et al. 2003; Brusa et al. 2005, 2007). Due to the high source densities of the ONIR catalogs and centroid errors in the X-ray positions, the simple method of searching for the nearest counterpart within a small radius could yield a nonnegligible number of false matches, usually to faint ONIR sources where the background source density is high. The likelihood-ratio technique (described in detail below) takes into account the positional accuracy of both the ONIR and Chandra catalogs and also the expected magnitude distribution of the counterparts. It assigns a reliability parameter to all the possible counterparts, and it mitigates the effect of false matches to faint ONIR sources.

\footnotetext{
${ }^{17}$ We used the entire GEMS catalog in the source-identification process for consistency. However, GEMS counterparts have a lower priority than GOODS-S counterparts when we select primary counterparts for the X-ray sources (see $\$ 2.3$

${ }^{18} \mathrm{We}$ also searched for SIMPLE $4.5 \mu \mathrm{m}$ counterparts, which yielded fewer identifications than the $3.6 \mu \mathrm{m}$ catalog.
}

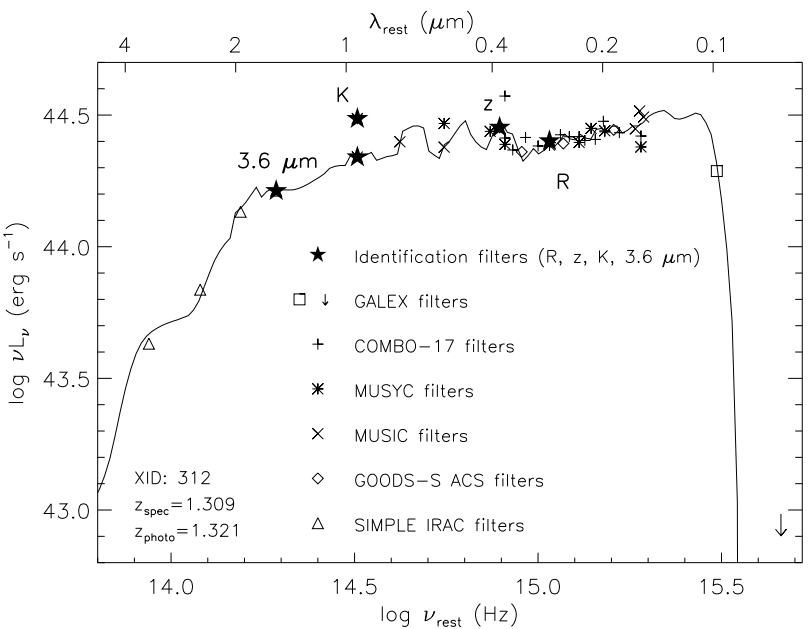

FIG. 1. - The SED of source 312 in L08 along with the selected best-fit SED template (a composite starburst/spiral galaxy template), as an example to illustrate the photometric bands used for source identification, the coverage of the broadband photometric data, and the photometric redshift fitting result. Error bars for the data points are smaller than or comparable to the symbol sizes and thus are not shown. Although this source is not detected in the radio band, the VLA $1.4 \mathrm{GHz}$ radio catalog was also used in the identification process (not shown in this plot). The source is not detected in the GALEX far-UV band, and the $1 \sigma$ upper limit is shown. There are 42 filters of photometric data displayed in the SED. Note that not all of the data points are used in the photometric redshift calculation (see $\$ 3.1$ for details). The discrepancy between some photometric data from different catalogs is probably caused by AGN variability.

For an ONIR candidate with a magnitude $m$ (at a given band) at an angular separation $r$ from a given X-ray source, ${ }^{19}$ the likelihood ratio is defined as the ratio of the probability of the ONIR object being the true counterpart of the X-ray source and the corresponding probability of the ONIR object being a background, unrelated object (e.g., Ciliegi et al. 2003),

$$
L R=\frac{q(m) f(r)}{n(m)},
$$

where $q(m)$ is the expected magnitude distribution of counterparts, $f(r)$ is the probability distribution function of the angular separation, and $n(m)$ is the surface density of background objects with magnitude $m$. We assume that the probability distribution of angular separations follows a Gaussian distribution (e.g., Ciliegi et al. 2003):

$$
f(r)=\frac{1}{2 \pi \sigma^{2}} \exp \left(\frac{-r^{2}}{2 \sigma^{2}}\right),
$$

with the standard deviation $\sigma=\sqrt{\sigma_{\mathrm{O}}^{2}+\sigma_{\mathrm{X}}^{2}}$, where $\sigma_{\mathrm{o}}$ and $\sigma_{\mathrm{X}}$ are the $1 \sigma$ positional errors of the ONIR and X-ray sources, respectively. The $1 \sigma$ positional errors of the X-ray sources were estimated by correlating X-ray sources to bright WFI $R$-band sources and then examining the positional offsets (see $\$ 3.3 .1$ of L08 for details). The $1 \sigma$ positional errors of the ONIR sources are either provided by the catalog or estimated from the distributions of positional offsets by matching the sources to the GOODS-S $z$-band catalog. The ONIR positional errors and X-ray positional errors are listed in Tables 1 and 2, respectively.

The magnitude-dependent surface density of the background sources, $n(m)$, was estimated using a sample of ONIR

\footnotetext{
${ }^{19}$ The $\mathrm{AB}$ magnitudes for radio sources were converted from the radio flux densities (e.g., Oke \& Gunn 1983), $m(\mathrm{AB})=-2.5 \log \left(f_{\nu}\right)-48.60$, where $f_{\nu}$ is the flux density in units of erg $\mathrm{cm}^{-2} \mathrm{~s}^{-1} \mathrm{~Hz}^{-1}$.
} 
sources that are at a angular distance between $5^{\prime \prime}$ and $30^{\prime \prime}$ from any of the X-ray sources. We chose sources inside such annular regions to exclude possible counterparts from the background calculation. The expected magnitude distribution of counterparts $q(m)$ is not directly observable. To estimate $q(m)$, we ran the matching procedure in an iterative way. The matching results from a previous run were used to calculate $q(m)$ for the next run. As a first guess, we selected all ONIR sources within $1^{\prime \prime}$ of any X-ray source, and we assume these ONIR objects are representative of the counterparts for the X-ray sources with a small contribution from coincident background sources. The number of sources in this sample is $N_{1}$, and the magnitude distribution of these sources is denoted as total $(m)$, which was then background subtracted to derive

$$
\operatorname{real}(m)=\operatorname{total}(m)-\pi r_{0}^{2} N_{\mathrm{X}} n(m),
$$

where $N_{\mathrm{X}}$ is the total number of X-ray sources in the relevant sample (see Col. 9 of Table 1), and $r_{0}=1^{\prime \prime}$. The background subtraction generally has a small effect (a few percent) on the resulting magnitude distribution. See Figure 2 for an example of $\operatorname{real}(m)$ and $n(m)$ in the GEMS $z$-band catalog. Due to the magnitude limits of the ONIR catalogs, we are only able to detect a fraction $Q$ of all the true counterparts $\left(Q=\int_{-\infty}^{m_{\lim }} q(m) d m\right)$. Thus the expected magnitude distribution of counterparts $q(m)$ is derived by normalizing real $(m)$ and then multiplying by $Q$ :

$$
q(m)=\frac{\operatorname{real}(m)}{\sum_{m} \operatorname{real}(m)} Q .
$$

The factor $Q$ was first set to $Q=N_{1} / N_{\mathrm{X}}$. The magnitude distributions, $q(m)$ and $n(m)$, are smoothed using a boxcar average (with width of one magnitude) and then used as input in the likelihood-ratio calculation. We note that source blending, which is most severe in the $3.6 \mu \mathrm{m}$ catalog, could affect the values of $q(m)$ and $n(m)$ by a tiny fraction and thus introduce small uncertainties in the matching results. However, as we choose counterparts carefully combining all seven catalogs (see $\$ 2.3$ ), this will not affect our final identifications.

Having computed the values of $q(m), f(r)$, and $n(m)$, we calculated $L R$ for all the ONIR sources within $5^{\prime \prime}$ of any of the $\mathrm{X}$-ray sources. We then chose a threshold value for $L R\left(L_{\mathrm{th}}\right)$ to discriminate between spurious and real counterparts. An ONIR source is considered to be a counterpart for an X-ray source if its $L R$ value exceeds $L_{\text {th }}$. In some cases, there will be more than one counterpart for an X-ray source within our $5^{\prime \prime}$ limiting radius. If the ratio between the highest and the second highest $L R$ values is greater than 5 , we keep only the counterpart with the highest $L R$ value ${ }^{20}$; otherwise, the X-ray source is considered to have multiple counterparts in this catalog. For each counterpart for an X-ray source identified this way, we calculate a reliability parameter, $R_{\mathrm{c}}$, which represents the probability of it being the correct identification:

$$
R_{\mathrm{c}}=\frac{L R}{\sum L R+(1-Q)},
$$

where the sum is over all the counterparts for this X-ray source.

\footnotetext{
${ }^{20}$ We believe those counterparts with much smaller $L R$ values are coincident matches; about 16 such unlikely counterparts were identified and removed in total, considering all the optical-to-IR catalogs. Because the final counterpart for an X-ray source is selected after combining the matching results for all the catalogs, the chance of removing a possible true counterpart here is negligible.
}

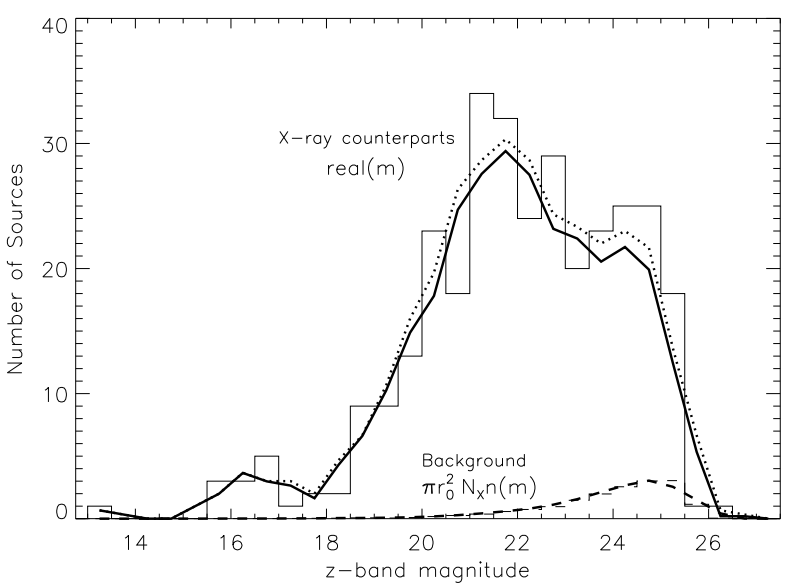

FIG. 2.- The expected magnitude distributions of the background sources (thin dashed histogram; normalized by a factor of $\pi r_{0}^{2} N_{\mathrm{X}}$ ) and counterparts (thin solid histogram) for the GEMS $z$-band catalog in the first trial run. The counterparts in the first trail run are GEMS sources within $1^{\prime \prime}$ of any X-ray source, and they were only used to calculate $q(m)$ and thus $L R$ (see $\sqrt[2.2]{2}$. The thick solid and dashed curves are the corresponding smoothed (with a boxcar average) distributions that represent real $(m)$ and $\pi r_{0}^{2} N_{\mathrm{X}} n(m)$ (see $\$ 2.2$ for details). The thick dotted curve shows the final smoothed magnitude distribution of the counterparts after three iterations. The magnitude distribution of the background sources peaks at fainter magnitude than the distribution of counterparts.

The choice of $L_{\text {th }}$ is not unique, and it depends on two factors: first, it should be small enough to avoid missing many real identifications; second, it should be large enough to prevent having many spurious identifications. In this paper, we followed the discussions in Ciliegi et al. (2003), Brusa et al. (2005, 2007), and Civano et al. (2010, in preparation), and we chose a $L_{\mathrm{th}}$ that maximizes the sum of sample completeness and reliability. The sample completeness parameter $(C)$ is defined as the ratio between the sum of the reliability of all the sources identified as counterparts and the total number of X-ray sources, $C=\sum R_{\mathrm{c}} / N_{\mathrm{X}}$. The sample reliability parameter $(R)$ is defined as the average of the reliabilities of all the sources identified as counterparts. For each run of the likelihood-ratio method, we tried several values of $L_{\mathrm{th}}$ and chose the one which maximizes the sum of $C$ and $R$.

After the first trial run of the likelihood-ratio procedure, we calculated $\operatorname{real}(m)$ as the magnitude distribution of all the sources identified as counterparts in the previous run, and we approximated the normalization factor $Q$ to be the completeness $C$ of the previous run. For each ONIR catalog, the likelihood-ratio procedure was run iteratively until the resulting values of $C$ and $R$ converged. This is a stable process with mild perturbations, and the results converge quickly, usually in $\approx 2-5$ iterations.

\subsection{Matching Results}

A summary of the likelihood-ratio matching parameters and results is shown in Table 1 We list the total number of X-ray sources that are within the coverage of the ONIR catalog $\left(N_{\mathrm{X}}\right)$, the number of X-ray sources identified with at least one ONIR counterpart $\left(N_{\text {ID }}\right)$, the number of X-ray sources without identifications $\left(N_{\text {NoID }}\right)$, and the number of X-ray sources identified with at least two ONIR counterparts $\left(N_{\text {Multi }}\right)$. We estimated the false-match probability of these identifications using a bootstrapping approach. We shifted the X-ray source coordinates by $5^{\prime \prime}-53^{\prime \prime}$ (with a $2^{\prime \prime}$ increment between shifts) in right ascension and declination respectively, and recorrelated the 
shifted sources with the ONIR sources using the likelihoodratio method. This was done for 100 trials, and the results were averaged to estimate the number of false matches. The expected number of false matches $\left(N_{\text {False }}\right)$ and the false-match probability for each catalog are shown in Table 1 The falsematch probabilities range from $\approx 1-9 \%$. The matching to the radio sources yields very secure counterparts, with less than one expected false match, largely due to the small background radio source density.

For each catalog, we calculated its counterpart recovery rate, defined as the expected number of true counterparts $\left(N_{\text {ID }}-N_{\text {False }}\right)$ divided by the number of X-ray sources $\left(N_{\mathrm{X}}\right)$, shown in Table 1 Compared to the completeness parameter $C$, the recovery rate parameter utilizes the bootstrapping results, and is a more appropriate estimate of the catalog completeness. The MUSIC NIR and SIMPLE IR catalogs have the highest recovery rates, while the VLA radio catalog recovers the fewest counterparts. We also calculated an $\mathrm{X}$-ray-to-ONIR fraction (X-O fraction) parameter, defined as the expected number of true counterparts $\left(N_{\text {ID }}-N_{\text {False }}\right)$ divided by the number of ONIR sources $\left(N_{\mathrm{o}}\right)$ in the catalog. The $\mathrm{X}-\mathrm{O}$ fraction indicates the degree of association between the X-ray and ONIR sources. Only $\approx 1-4 \%$ of the opti$\mathrm{cal} / \mathrm{NIR} / \mathrm{IR}$ sources are X-ray sources, whereas $\approx 28 \%$ of the radio sources are detected by Chandra. A larger X-O fraction will lead to more reliable source matching results because of the lower background source density. Both the recovery rate and $\mathrm{X}-\mathrm{O}$ fraction parameters are dependent on the catalog depth.

Given the excellent radio-source positional accuracy and the secure matching results, we can investigate the accuracy of the X-ray source positions using the information for the radio counterparts. ${ }^{21}$ In Figure 3 , we show the positional offset between the $94 \mathrm{X}$-ray sources and their radio counterparts versus off-axis angle. The median offset is $0.4^{\prime \prime}$, and there are clear off-axis angle and source-count dependencies. The off-axis angle dependence is mainly due to the degradation of the Chandra PSF at large off-axis angles, while the count dependence is due to the difficulty of finding the centroid of a faint X-ray source. Although the results are comparable to the L08 study of the positional accuracy based on the matching to the WFI $R$-band sources (see $\$ 3.3 .1$ of L08), Figure 3 should be considered more representative of the typical X-ray positional accuracy, as the radio catalog provides the most-reliable $\mathrm{X}$-ray source identification.

Twenty of the $462 \mathrm{X}$-ray sources do not have a counterpart in any of the ONIR databases, based on the likelihood-ratio matching to all seven catalogs. Four (XIDs 72, 165, 234, and 396 ) of these 20 sources have a faint ONIR source (a $z$-band source in each case, with $z=25.3-26.7$ ) within $\approx 0.7-1.2^{\prime \prime}$ of the X-ray position; these ONIR sources were not selected by the likelihood-ratio matching due to their small likelihood ratios $\left(L R<L_{\mathrm{th}}\right)$. However, given the relatively small positional offsets of these four sources, the lack of any other apparent counterparts, and the fact that the likelihood-ratio matching is a statistical method and cannot possibly identify all the counterparts, we manually assign them as the counterparts of the relevant X-ray sources (see Col. 13 of Table 2). Thus 446

\footnotetext{
21 Three of the radio counterparts (those of sources 200, 364, and 368) are identified as the lobe components of multi-component radio sources by Kellermann et al. (2008). However, the radio counterparts of sources 364 and 368 are probably two interacting galaxies based on optical imaging and spectroscopic results (e.g., Mainieri et al. 2008); these two radio sources are blended in Kellermann et al. (2008), but well resolved in Miller et al. (2008).
}

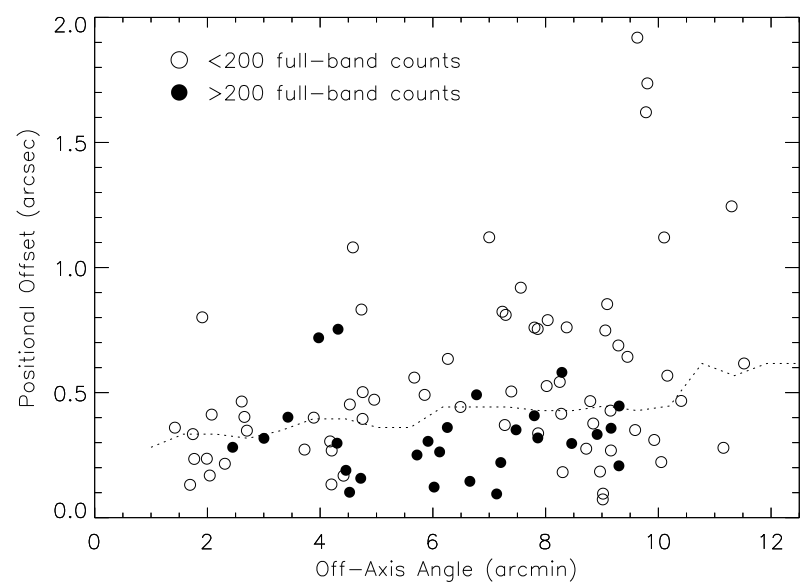

FIG. 3.- Positional offset vs. off-axis angle for sources in the main $\mathrm{X}$-ray source catalog of L08 that were matched to the VLA radio sources (Miller et al. 2008). The open circles represent Chandra sources with $<200$ full-band counts, and the solid dots are Chandra sources with $\geq 200$ full-band counts. The dotted curve shows the running median of all sources in bins of $2^{\prime}$. The three outliers on the top right corner of the plot are faint X-ray sources with large positional errors $\left(\sigma_{\mathrm{X}} \approx 0.5^{\prime \prime}-0.7^{\prime \prime}\right)$.

$\mathrm{X}$-ray sources have identifications, including these four additional likely cases. Note that the false-match probabilities in Table 1 do not apply to these four sources. For the remaining 16 sources without identifications, there is no ONIR source within $1.3^{\prime \prime}$. As the ONIR catalogs we used were all generated using red bands, we also visually inspected images in bluer bands (e.g., $U, B$, and $V$ ) at the positions of these 16 sources, and verified that we did not miss any apparent blue counterparts for them. Eight of these unidentified sources are probably related to some other objects (e.g., off-nuclear sources; see Col. 13 of Table 2), and the majority of the other eight are likely to be spurious X-ray sources (see discussion in \$4.1).

Twenty of the sources only have a SIMPLE counterpart; however, eight of these SIMPLE counterparts have a corresponding optical/NIR source nearby (within $0.8^{\prime \prime}$ ), and they are likely to be the same source. Therefore we used the more accurate optical/NIR positions for these eight counterparts (see Col. 13 of Table 2).

Some X-ray sources could have multiple counterparts, either from one ONIR catalog as a result of the likelihoodratio matching (e.g., see the $N_{\text {Multi }}$ parameter in Table 1), or from different optical-to-radio catalogs. There are $12 \mathrm{X}$-ray sources with multiple counterparts in at least one of the ONIR catalogs; none of them has more than two counterparts in one band. For the remaining 434 sources, we consider an X-ray source to have multiple counterparts if the angular distance between two of the counterparts in different bands exceeds $3 \sqrt{\sigma_{1}^{2}+\sigma_{2}^{2}}$ (so that they appear to be different sources), where $\sigma_{1}$ and $\sigma_{2}$ are the positional errors in the relevant ONIR catalogs. There are 60 such sources; five of them have three counterparts. In all, $72 \mathrm{X}$-ray sources have multiple counterparts (with the other $374 \mathrm{X}$-ray sources having a unique counterpart), and they are flagged in Column 12 of Table 2 .

For each of the $446 \mathrm{X}$-ray sources with an identification, we visually inspected all its counterparts in the appropriate bands, and chose a primary one. The position of this source is the most probable position of the counterpart. We chose the primary counterpart from, in order of priority, the VLA, GOODS-S, GEMS, MUSIC, WFI, MUSYC, or SIMPLE catalog. This order is chosen based on several related 
factors: the positional accuracy, angular resolution (to minimize any blending effects), false-match probability, and catalog depth. ${ }^{22}$ There are a few exceptions: (1) if the optical counterpart is clearly extended, i.e., a nearby galaxy, we select the optical position instead of the VLA radio position because the radio emission could arise from an off-nuclear structure (7 cases); (2) if the optical counterpart is bright and significant, while the radio counterpart is detected at a smaller than $7 \sigma$ significance, we prefer the optical position (4 cases); (3) when the counterpart from a lower-priority catalog is much brighter and better resolved, or it has a significantly larger reliability parameter, we prefer this source as the primary counterpart (5 cases). The final number of primary counterparts selected in each catalog $\left(N_{\text {Pri }}\right)$ is listed in Table 1. The mean false-match probability for the 442 primary counterparts (excluding the the four additional likely counterparts) is then estimated by taking the average of the false-match probabilities of individual catalogs weighted by the number of primary counterparts in each catalog. The mean false-match probability is $\approx 6.2 \%$, and it represents the identification reliability for the entire Xray sample (excluding the the four additional likely counterparts). The mean false-match probability also has slight offaxis angle and source-count dependencies (due to its relation to the $\mathrm{X}$-ray positional accuracy); e.g., it is $\approx 6.0 \%$ for sources within $4^{\prime}, \approx 7.0 \%$ at $>8^{\prime}, \approx 9.8 \%$ for sources with $<30$ fullband counts, and $\approx 5.8 \%$ for $>30$ full-band counts.

A list of the primary counterparts for the $446 \mathrm{X}$-ray sources (out of 462 total sources) is presented in Table 2, with the details of the columns given below; we also list all the secondary counterparts (for sources with multiple counterparts) in Table 3 with a similar format.

- Column (1): the X-ray source ID number, which is the same as the source number in the main catalog of L08.

- Column (2): The $1 \sigma$ X-ray source positional error $\sigma_{\mathrm{X}}$.

- Column (3): the ONIR ID number of the primary counterpart, taken from the corresponding ONIR catalog (see Col. 10). For the 16 X-ray sources that lack a counterpart, the value is set to " 0 ".

- Columns (4) and (5): the ONIR J2000 right ascension and declination of the counterpart, respectively. This position is taken from the corresponding ONIR catalog (see Col. 10). X-ray sources that do not have a counterpart have this column set to " 0 ".

- Columns (6): the approximate $1 \sigma$ positional error of the counterpart, in units of arcseconds, taken from Column 6 of Table 1

- Column (7): the angular distance between the X-ray source and its ONIR counterpart, in units of arcseconds, set to " 0 " if there is no counterpart.

- Column (8): the angular distance divided by the quadratic sum of the positional errors $\left(\sigma=\sqrt{\sigma_{\mathrm{O}}^{2}+\sigma_{\mathrm{X}}^{2}}\right)$, i.e., the angular distance in units of $\sigma$.

- Column (9): the reliability parameter of this counterpart (see Eq. 5). X-ray sources that do not have a counterpart have a value of " 0 ".

22 Although we adopted the same positional error for some catalogs (e.g., GOODS-S and GEMS), the deeper catalogs (e.g., GOODS-S) can in principle provide slightly better positions for the counterparts.
- Column (10): the ONIR catalog from which the primary counterpart was selected. X-ray sources that do not have a counterpart have this column set to "...".

- Column (11): the ONIR magnitude of the primary counterpart (see Col. 10 for the band in which the magnitude was measured). X-ray sources that do not have a counterpart have this column set to " 0 ".

- Column (12): a flag on whether the X-ray source has multiple ONIR counterparts, set to " 1 " for true and " 0 " for false.

- Column (13): notes on some special sources, including the $16 \mathrm{X}$-ray sources that do not have ONIR identifications (cases $a-e$ ) and a few other sources (cases $f-h$ ). (a) NoID: there is no ONIR source within $\approx 1.3-3.6^{\prime \prime}$ of the X-ray source position, and it is likely that this X-ray source does not have an ONIR counterpart. There are eight such sources. (b) Edge: this source lies at the edge of the $\approx 2$ Ms CDF-S field, with a large PSF size. The X-ray photons could come from any of a number of galaxies in the vicinity. There is one such source. $(c)$ Cluster: the X-ray source may be associated with the diffuse emission from a galaxy group or cluster. Galaxy groups and clusters in the $\approx 2 \mathrm{Ms} C D F-S$ are identified through a wavelet detection of extended X-ray emission (Finoguenov et al. 2010, in preparation). There are four such sources, which are generally faint and weakly detected in the X-ray. (d) Star: the source is only detected in the soft X-ray band, and is $2.7^{\prime \prime}$ away from a bright $(R=16)$ star. The X-ray photons are probably largely from the star, and the X-ray position was not correctly determined by WAVDETECT due to the contamination of another possible faint X-ray source nearby. There is one such source. (e) ULX: this is an off-nuclear Xray source associated with a nearby galaxy. There are two such sources. One of them (source 301) is reported in Lehmer et al. (2006). (f) Blend: the primary counterpart is blended with an adjacent source, and the position of the counterpart is poorly determined (positional uncertainty $\left.\approx 1-2^{\prime \prime}\right)$. There is one such source, and the primary counterpart is from the SIMPLE catalog (and there are no detections of this source at shorter wavelengths). ( $g$ ) Faint: there is at least one faint ONIR source within $\approx 0.7-1.2^{\prime \prime}$ of the X-ray source position. The likelihood ratio parameter of this ONIR source is below the threshold, but we manually assigned it to be the counterpart. There are four such sources. $(h)$ Position: the source only has a counterpart from the SIMPLE catalog. However, the SIMPLE counterpart likely coincides with a nearby optical/NIR source, and we choose this optical/NIR source as the primary counterpart because of its more accurate position (see above). There are eight such sources.

The locations of the 446 identified and 16 unidentified $\mathrm{X}$-ray sources in the CDF-S region are shown in Figure 4 along with the coverages of the GOODS-S and MUSIC catalogs, which are the only two catalogs that do not cover the entire CDF-S. For the 446 sources with an ONIR identification, the mean and median positional offsets between the $\mathrm{X}$-ray sources and their primary counterparts are $0.41^{\prime \prime}$ and $0.32^{\prime \prime}$, respectively. The maximum offset is $\approx 1.9^{\prime \prime}$. Several X-ray sources have large positional offsets $\left(\gtrsim 1.5^{\prime \prime}\right)$ to their 


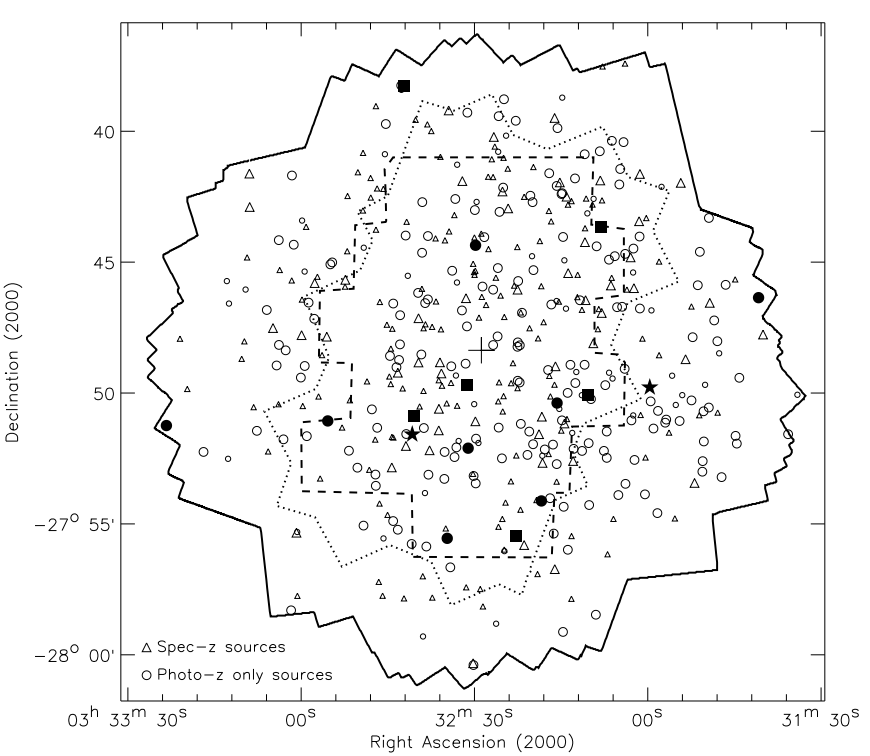

FIG. 4.- Positions of the 446 identified (open symbols) and 16 unidentified (filled symbols) X-ray sources. The open triangles and circles represent sources with spectroscopic and photometric redshifts, respectively, and small (large) symbols indicate redshifts smaller (larger) than 1.3 (see 3.3 for details about redshifts). The eight filled circles represent sources that are probably related to a star, galaxy groups and clusters, off-nuclear sources, or any of a number of galaxies at the edge of the CDF-S field (see Col. 13 of Table 2. The six filled squares and two filled stars represent the "NoID" (Col. 13 of Table 2. sources that were detected at a WAVDETECT false-positive threshold of $1 \times 10^{-6}$ and $1 \times 10^{-8}$, respectively (see $\$ 4.1$ for discussion). The solid, dotted, and dashed curves outline the regions covered by the $\approx 2 \mathrm{Ms}$ CDF-S, GOODS-S, and MUSIC surveys, respectively. The cross near the center indicates the average aim point of the CDF-S.

primary counterparts. These counterparts are either selected from the SIMPLE catalog (less-accurate positions), VLA catalog (low background source density), or are relatively bright, so that the likelihood ratio could still exceed the threshold at large ONIR-X-ray separations (see \$2.2). Figure 5 shows the distributions of the positional offsets in four bins of different X-ray positional uncertainties. The positional offsets are generally smaller than or comparable to the $\approx 85 \% \mathrm{X}$-ray positional uncertainties (dashed lines in Fig. 55) for most sources.

\subsection{Comparison with the Error-Circle Matching Method}

To check the efficiency of the likelihood-ratio technique, we compared the matching results above to those derived from the simple error-circle method described in $\$ 3.3 .1$ of L08. ${ }^{23}$ For the WFI $R$-band, L08 gives 347 matches with an expected false-match probability of $12 \%$ (305 expected true matches), while here we have 344 matches with a false-match probability of $9 \%$ (312 expected true matches). As expected, the likelihood-ratio technique provides more reliable matches $(\approx 7$ more sources using our approach). Between the 347 counterparts from the error-circle method and the 344 counterparts from the likelihood-ratio method (if a source has multiple counterparts, we chose the one with the highest reliability parameter), 11 are different matches, six of which have a

${ }^{23}$ In L08, we searched for the closest optical source within a matching radius of $1.5 \sqrt{\Delta_{\mathrm{X}}^{2}+\sigma_{\mathrm{o}}^{2}}$, where $\Delta_{\mathrm{X}}$ is the $\approx 85 \%$ confidence-level X-ray positional error.

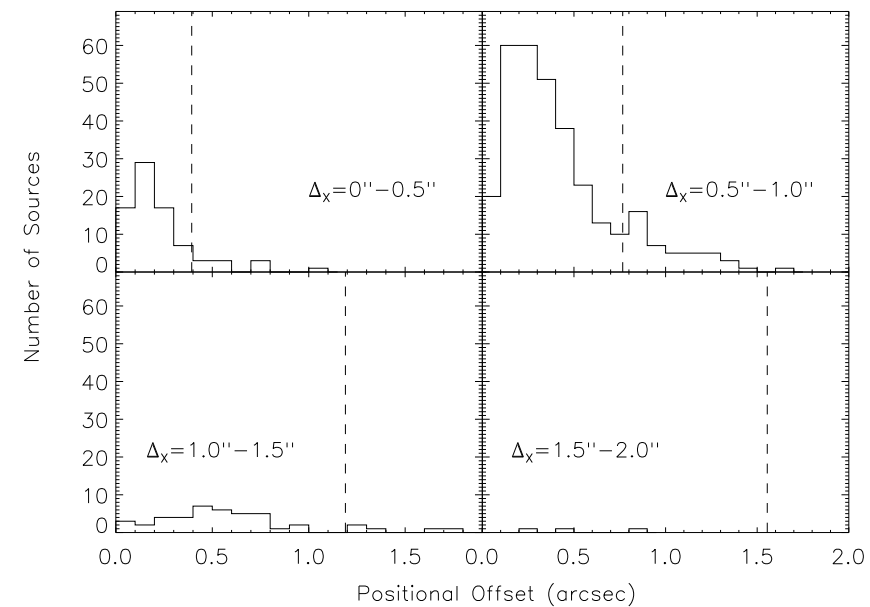

FIG. 5.- Histograms showing the distributions of the positional offset between an X-ray source and its primary counterpart. X-ray sources are divided into four bins based on their $\approx 85 \%$ positional uncertainties $\left(\Delta_{X}\right)$ given in L08: $0^{\prime \prime}-0 .{ }^{\prime \prime} 5,0{ }^{\prime \prime} 5-1$.' $^{\prime} 0,1{ }^{\prime \prime} 0-1{ }^{\prime \prime} 5$, and 1 . $^{\prime \prime} 5-2$.' $^{\prime \prime} 0$. The vertical dashed lines indicate the median positional uncertainties (L08) for X-ray sources in each bin. The positional offsets are generally smaller than or comparable to the $\mathrm{X}$-ray $\approx 85 \%$ positional uncertainties for most of the sources.

likelihood-ratio counterpart with magnitude of $R>25.5$.

We studied the relation between the false-match probability and catalog depth by setting different $R$-band magnitude limits. The results are shown in Figure 6, which indicate that the likelihood-ratio method outperforms the error-circle method at faint magnitudes, and this is consistent with the expectation that the likelihood-ratio method can guard against false matches to faint sources. Indeed, the six different counterparts with $R>25.5$ derived from the two methods contribute to $20 \%$ of the total population of $30 R>25.5$ counterparts for the entire X-ray sample (see also Brusa et al. 2009).

From the cumulative distributions of false-match probabilities shown in Figure 6, we calculated the differential values at several $R$-band magnitudes. For the likelihood-ratio (error-circle) method, the false-match probabilities are $\approx 7 \%$, $16 \%, 20 \%$, and $24 \%(\approx 7 \%, 21 \%, 28 \%$, and $37 \%)$ at $R=24$, 25,26 , and 27 , respectively, showing the challenge of deriving secure matches at faint optical magnitudes, even with the likelihood-ratio technique.

As a further test, we performed the matching with the deeper GOODS-S $z$-band catalog using the error-circle method. There are 266 matches and a $20 \%$ false-match probability, while the likelihood-ratio method yields 259 matches and a $9 \%$ false-match probability. 14 matches are different from the error-circle results. Ten of these different matches have $z>25.5$, contributing to $\approx 30 \%$ of all the counterparts with $z>25.5$. Note here that the matching results and the false-match probability of the error-circle method depend on the choice of the matching radius. Using the same matching radius as that for the WFI $R$-band might not be optimal for the GOODS-S $z$-band. It is laborious to determine the optimal matching radius for the error-circle method, i.e., the matching radius that provides a large number of counterparts without introducing too many false matches. ${ }^{24}$ On the other hand, the likelihood-ratio method can perform a similar optimization (in terms of the completeness and reliability parameters)

24 We tried several other matching radii and none of them can provide better results than the likelihood-ratio method. For example, a matching radius of $1.1 \sqrt{\Delta_{X}^{2}+\sigma_{\mathrm{o}}^{2}}\left(1.3 \sqrt{\Delta_{\mathrm{X}}^{2}+\sigma_{\mathrm{o}}^{2}}\right)$ resulted in 252 (261) matches with a $13 \%$ $(16 \%)$ false-match probability. 


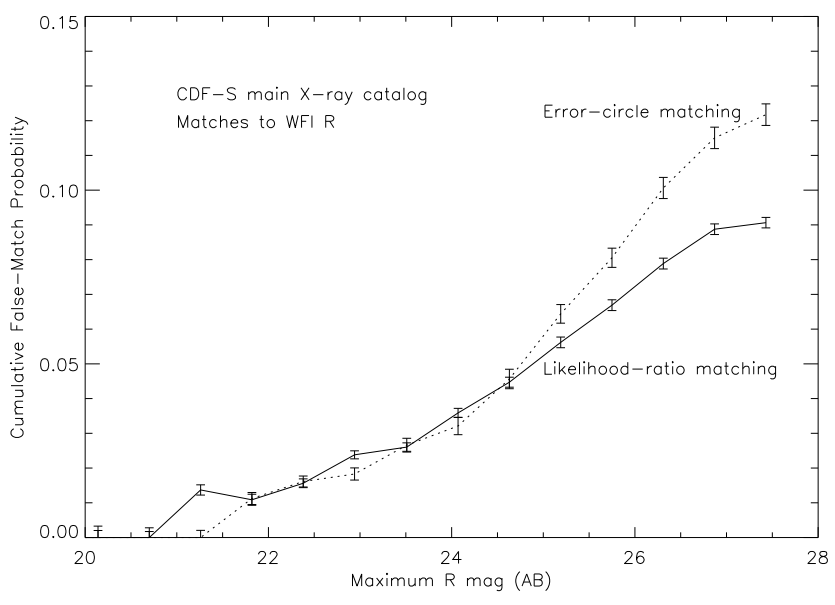

FIG. 6.- Cumulative false-match probability as a function of the $R$-band limiting magnitude for the error-circle method (dotted curve) and the likelihood-ratio method (solid curve). The false-match probabilities are estimated using the bootstrapping technique described in $\$ 2.3$ Error bars indicate the errors in the mean values of the false-match probabilities at different magnitude limits. The corresponding differential false-match probabilities for the likelihood-ratio (error-circle) method are $\approx 7 \%, 16 \%, 20 \%$, and $24 \%(\approx 7 \%, 21 \%, 28 \%$, and $37 \%)$ at $R=24,25,26$, and 27 , respectively. The likelihood-ratio method yields more reliable matches at faint magnitudes. The error-circle method appears to have a generally smaller false-match probability at bright magnitudes $(R \lesssim 23)$; however, it also gives a smaller number of matches compared to the likelihood-ratio method, and the numbers of expected true matches from these two methods are comparable.

automatically and more carefully, and is thus more robust.

We conclude that the likelihood-ratio technique can provide much more reliable matches than the error-circle method at faint optical magnitudes (e.g., $R \gtrsim 25$ ). The resulting matches may differ by $\approx 20 \%$ in this regime, where we expect to find some of the most interesting objects in deep X-ray surveys, such as heavily obscured AGNs and AGNs at very high redshifts. When matching to shallower catalogs with low source densities, the likelihood-ratio and error-circle methods will produce similar results with competitive reliabilities; however, the multiple candidates and reliability parameters provided by the likelihood-ratio matching are still useful and convenient when considering multiwavelength identifications.

\section{PHOTOMETRIC REDSHIFTS}

The CDF-S area has been targeted intensively by photometric and spectroscopic surveys, allowing us to determine robustly the photometric redshifts (hereafter, photo-z's) of the $\mathrm{X}$-ray sources. Compared to previous studies of the photo-z's of the optical sources (e.g., Wolf et al. 2004) or X-ray sources (e.g., Zheng et al. 2004) in the CDF-S region, our photo-Z work here has the following features and advantages: (1) We are utilizing the X-ray sources in the current $\approx 2 \mathrm{Ms}$ Chandra catalog, with the most reliable counterpart information available derived using the likelihood-ratio matching method. (2) The most recent UV-to-IR deep surveys provide an unprecedented multiwavelength data set for photo-z calculation; the Spitzer, GEMS HST, and GALEX UV data have been used for the first time in the photo- $\mathrm{Z}$ calculation for the CDF-S X-ray sources. As we are dealing with a relatively small sample of sources, we were able to carry out source deblending carefully and obtain the best-ever photometric data for them (see $\$ 3.1$. The likelihood-ratio method is used when matching the primary counterpart positions (Columns 4 and 5 in Table 2) to the photometric catalogs, and the false-match probabilities are small $(\lesssim 4 \%)$. Details regarding the multiwavelength data are described below. (3) A collection of up-to-date secure spectroscopic redshifts (hereafter spec-z's) allows better calibration of the SED templates and a fair assessment of the photo-z quality.

Of the 446 identified X-ray sources, 220 have secure spec-z's. We collected secure spec-z's ( $\gtrsim 95 \%$ confidence level, with several secure spectral features) from the following catalogs: Le Fèvre et al. (2004), Szokoly et al. (2004), Zheng et al. (2004), Mignoli et al. (2005), Ravikumar et al. (2007), Vanzella et al. (2008), Popesso et al. (2009), and Silverman et al. (2010, in preparation). A matching radius of $0.5^{\prime \prime}$ was used when matching the primary counterparts to the redshift catalogs. ${ }^{25}$ A bootstrapping test similar to that in $\S 2.3$ indicates a false-match probability of $0.8 \%$ for these spec-z's. We refer to these 220 sources with secure spec-z's as the spectroscopic sample. Of the other 226 sources, six are identified as stars (see details below in $\$ 3.2$ ), and we refer to the remaining 220 sources as the photometric sample. Both the spectroscopic and photometric samples have 220 X-ray sources, and their photo-z's have been computed. According to the X-ray and optical property analysis in Bauer et al. (2004) and Xue et al. (2010, in preparation), more than $\approx 75 \%$ of these sources are AGNs (see also §3.3), while the others appear to be starburst or normal galaxies.

\subsection{Multiwavelength Photometric Data}

We constructed a photometric catalog for the 446 identified X-ray sources including up to 42 bands of UV-to-IR data (up to 35 bands are used in the photo-z calculation for a given source; see below), derived from a number of publicly available catalogs. For a source undetected in a given band, if the non-detection is not caused by the possible blending with a nearby source, we estimated its $1 \sigma$ magnitude upper limit, using the background rms maps generated by SEXTRACTOR (Bertin \& Arnouts 1996). The $1 \sigma$ instead of the more conservative $3 \sigma$ upper limits were calculated because these upper limits are used later in the photo-z calculation (see \$3.2). Upper limits are not available for the COMBO-17 and MUSIC bands, and we calculated only the $z$-band upper limits among the HST ACS bands. We prioritized the catalogs as an indication of our preference for using the data from these catalogs, with a highest priority of 5 and lowest 1 . The priorities are determined considering the spatial resolution and number of bands available (for the consistency in colors, e.g., the MUSIC $U$-band has a higher priority than the VIMOS $U$-band). Details on the catalogs are given below.

1. The GALEX UV catalog from GALEX Data Release 4, which includes the near-UV (NUV, $\left.\lambda_{\text {eff }}=2267 \AA\right)$ and far-UV (FUV, $\lambda_{\text {eff }}=1516 \AA$ ) data. ${ }^{26}$ The $5 \sigma$ limiting AB magnitudes for the NUV and FUV bands are 26.0 and 26.4, respectively. This catalog has a priority of 1 .

2. The GOODS-S deep VIMOS $U$-band catalog (Nonino et al. 2009), with a $1 \sigma$ limiting AB magnitude of 29.8. This catalog has a priority of 1 .

3. The COMBO-17 optical catalog $(U, B, V, R, I$, plus 12 narrow-band filters; Wolf et al. 2004, 2008). The $10 \sigma$ limiting $\mathrm{AB}$ magnitudes for the $U, B, V, R$, and $I$ bands

\footnotetext{
25 We cannot apply the likelihood-ratio method described in $\$ 2.2$ to match the primary counterparts to the optical sources with secure redshifts, as these sources are not from well-defined and flux-limited catalogs.

26 http://galex.stsci.edu/GR4/.
} 
are $24.7,25.0,24.5,25.6$, and 23.0, respectively. This catalog has a priority of 3 .

4. The MUSYC $B V R$-detected optical catalog $(U, B, o 3$, $V, R, I$, and $z$ filters; Gawiser et al. 2006), and the MUSYC $K$-detected optical/NIR catalog $(B, V, R, I$, $z, J$, and $K$ filters; Taylor et al. 2009). The $5 \sigma$ limiting $\mathrm{AB}$ magnitudes for the $J$ and $K$ bands are 23.1 and 22.4 , respectively. These catalogs have priorities of 2 .

5. The GOODS-S MUSIC optical/NIR/IR catalog $\left(U_{35}\right.$, $U_{38}, U, F 435 W, F 606 W, F 775 W, F 850 L P, J, H, K$, and IRAC filters; Grazian et al. 2006). The $90 \%$ limiting $\mathrm{AB}$ magnitudes for the $J, H$, and $K$ bands are $24.5,24.3$, and 23.8 , respectively The $U$-band filter is the same as that used in the GOODS-S deep VIMOS $U$-band catalog. This catalog has a priority of 3 .

6 . The WFI $R$-band catalog (see $\S 2$ of Giavalisco et al. 2004), with a $5 \sigma$ limiting $\mathrm{AB}$ magnitude of 27.3. The $R$-band filter is the same as that used in the COMBO-17 catalog. This catalog has a priority of 2 .

7. The GOODS-S HST ACS $B, V, i$, and $z$-band $(F 435 W$, $F 606 \mathrm{~W}, F 775 \mathrm{~W}$, and $F 850 L P$ filters) optical catalog, with $5 \sigma$ limiting $\mathrm{AB}$ magnitudes of $29.1,29.1,28.5$ and 28.2 , respectively. This catalog has a priority of 5 .

8. The GEMS $V$ and $z$-band $(F 606 W$ and $F 850 L P$ ) cata$\log ($ Caldwell et al. 2008) $),{ }^{27}$ with $5 \sigma$ limiting AB magnitudes of 28.5 and 27.3, respectively. This catalog has a priority of 4 .

9. The SIMPLE IRAC $3.6 \mu \mathrm{m}, 4.5 \mu \mathrm{m}, 5.8 \mu \mathrm{m}$, and $8.0 \mu \mathrm{m}$ IR catalog (Damen et al. 2010), with $5 \sigma$ limiting $\mathrm{AB}$ magnitudes of 23.8, 23.7, 22.0, and 21.8, respectively. This catalog has a priority of 1 .

To collect photometric data from these catalogs for the $\mathrm{X}$-ray sources, we matched the primary counterparts of the $\mathrm{X}$-ray sources to the UV, optical/NIR, and IR catalogs using the same likelihood-ratio method described in $\$ 2.2$. The bootstrapping exercises gave small false-match probabilities $(\lesssim 4 \%)$. The aperture-corrected total magnitudes were extracted from the catalogs for each matched source. Figure 1 shows the SED of source 312 as an example of the resulting multiwavelength photometric data.

Not all the data are used in the photo-z calculation. We ignored the data if the errors are larger than one magnitude. When detections in the same filter are present in different catalogs, we chose the data from the catalog with the highest priority. When a source has both MUSYC and COMBO-17 detections, the MUSYC optical data (except for the $o 3$ and $z$ bands) were ignored, as there are a larger number of filters in the COMBO-17 data. When a source has both MUSYC and MUSIC NIR detections, we neglected the MUSYC NIR data. Therefore, any individual source can have up to 35 filters of photometric data that are used for deriving the photoz. We prefer the IRAC data in the GOODS-S MUSIC catalog because the IRAC measurements are less affected by possible source-blending problems. In cases when photometric data in two similar filters (e.g., GOODS-S MUSIC $U$-band and MUSYC $U$-band) differ by more than 0.5 magnitudes

${ }^{27}$ See http://www.mpia-hd.mpg.de/GEMS/gems.htm for the $V$-band data. (which may be caused by source variability or blending), we used only the data with the higher priority. For about ten GEMS sources, the $z$ and $V$-band magnitudes are $\gtrsim 1 \mathrm{mag}$ nitude fainter than the MUSYC $z$ and $V$-band data. We derived aperture photometry (with a $0.7^{\prime \prime}$ aperture radius) results using the GEMS science images, and they agree with the MUSYC photometry data. These sources appear to have some low-surface-brightness emission, and the GEMS cata$\log$ underestimates their brightness. Therefore we ignored the GEMS data for these sources. Upper-limit information was only used when there is no detection in a given band and all other similar bands.

As we already know the presence of the X-ray sources and their primary counterparts, we can search for their detections in the optical/NIR images at low significance levels with less worry about false detections. Therefore, we ran SEXTRACTOR on the WFI $R$-band and MUSYC optical/NIR images with various detection significances down to $1 \sigma$; we did not search the GOODS-S and GEMS optical images because the corresponding catalogs are already very complete. On average, only a few additional detections were found in each band, and we visually examined the images to verify their validity.

To reduce the effects of source blending, we inspected each source and corrected its photometric data if these are affected by blending. About $10 \%$ of the sources in the IRAC images and a few percent in the optical/NIR are blended with adjacent sources, and they are usually resolved in the deep HST images, i.e., in the GOODS-S or GEMS $z$ band. We deblended these sources by calculating the flux ratios using the pixel values at the source positions. In some cases when there is only mild blending in the IRAC images or the blended sources are not detected in the $z$ band, we manually extracted the aperture fluxes by centering on the correct source positions (positions of the primary counterparts) and using an aperture radius of $1.5^{\prime \prime}$; the fluxes were then aperture corrected to give the total flux. For the one case where the primary counterpart is blended (source 22), we centered on the X-ray position to derive the IRAC fluxes. We ignored the UV data if the source suffers from blending, because the UV image resolution is not as good as those for the optical/NIR/IR images.

The final photometric catalog is presented in Table $4^{28}$ Rows 2-463 of Table 4list the source X-ray IDs (Column 1) as well as the photometric data, data errors, and data flags in each band: the GALEX bands (Columns 2-7), VIMOS $U$ band (Columns 8-10), COMBO-17 bands (Columns 11-61), MUSYC bands (Columns 62-103), MUSIC bands (Columns 104-145), WFI $R$ band (Columns 146-148), GOODS$S$ ACS bands (Columns 149-160), GEMS ACS bands (Columns 161-166), SIMPLE IRAC bands (Columns 167178), and VLA 1.4 GHz band (Columns 179-181). The radio data are used only in the X-ray source identification process, and are not used in the photo-z calculation. All the photometric data are given in AB magnitudes, and are set to "99" if not available; upper limits are indicated by the "-" sign. A value of " 1 " for the data flag indicates that the data point was used in the photo- $z$ calculation, " 0 " means that the data point was not used or not available, and " -1 " means that the data point is probably problematic, either being blended or disagreeing with other data. Row 1 of Table 4 provides, for the identified extragalactic sources, the median magnitude (in the magnitude column), normalized median absolute devia-

28 A FITS file containing Table 4 (without the first row) is available at http://www.astro.psu.edu/ niel/cdfs/cdfs-chandra.html 


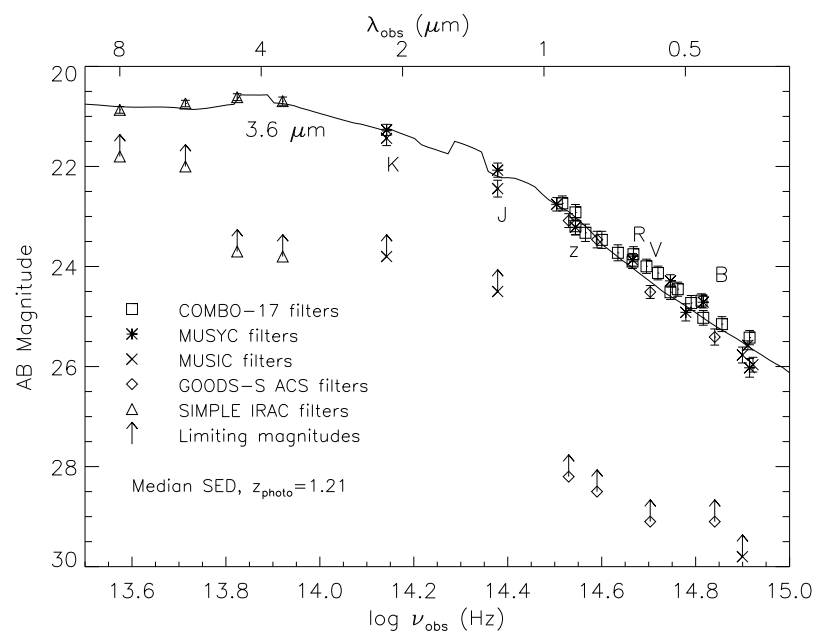

FIG. 7.- The observed median SED for the CDF-S X-ray sources and its best-fit SED template (a hybrid type 2 AGN template). Data in the same filter are combined (e.g., GOODS-S $z$ and GEMS $z$, MUSIC $U$ and VIMOS $U$ ). The NUV, FUV, and MUSIC $H$ bands are not shown as the median magnitudes were calculated using only the detected sources in these bands, and thus the SED consists of 39 bands of data. The MUSIC $J, K$, and GOODS$\mathrm{S} B$ and $i$-band data only represent $\mathrm{X}$-ray sources in the GOODS-S region. The errors for the median magnitudes are estimated using $N M A D / \sqrt{N_{\text {med }}}$. The limiting magnitudes for some of the deep optical-to-IR bands are also shown. This fit should not be interpreted physically as sources across a broad range of redshift are included.

tion (NMAD; in the magnitude-error column), and number of X-ray sources used to derive the median magnitude $\left(N_{\text {med }}\right.$; in the data flag column) for each band. ${ }^{29}$ The median magnitude in a given band was calculated based on the magnitudes of all the 440 sources (after excluding the 16 unidentified sources and 6 stars), and for undetected sources, the limiting magnitude of that band was used. ${ }^{30}$ There are some exceptions: for the GOODS-S and MUSIC catalogs, we used only X-ray sources within the coverage of these catalogs; for the NUV, FUV, and VLA $1.4 \mathrm{GHz}$ bands, in which less than half of the X-ray sources are detected, and for the MUSIC $U$ and $H$ bands, which cover only a portion of the MUSIC field, we used only detected sources to calculate the median magnitudes. The data in Row 1 indicate how bright a typical CDF-S X-ray source is in each band; note that the median values in some bands do not sample the entire CDF-S X-ray source population. The SED derived from the median magnitudes and its best-fit template are shown in Figure 7 (see details about the SED template library and photo-z fitting procedure in $\$ 3.2$. This fit should not be interpreted physically as sources across a broad range of redshift are included.

Figure 8, shows the distributions of the number of filters in which the source is detected, and the number of filters used in the photo-z calculation (including upper limit data). The maximum values of these two filter numbers are both 35 , and the minimum values are 3 and 7, respectively. We also show in Figure $8 \mathrm{~b}$ that brighter $R$-band sources tend to have better multiwavelength coverage with a larger number of filters. ${ }^{31}$

\footnotetext{
29 The normalized median absolute deviation is a robust measure of the spread of the magnitudes $(\sigma)$ in a given band, defined as $N M A D=1.48 \times$ median $(|m(\mathrm{AB})-\operatorname{median}(m(\mathrm{AB}))|)$ (e.g., Maronna et al. 2006).

30 The median magnitude and normalized median absolute deviation are stable parameters in general, and are not significantly affected by the choice of using the limiting magnitude or the individual magnitude upper limits for undetected sources, as long as more than half of the sources are detected.

${ }^{31}$ For some sources that do not have a WFI $R$-band detection, we used the MUSYC $R$-band magnitudes if available. The MUSYC survey searched for sources in the $B, V, R$, and $K$ bands and thus provides additional detections.
}

Sources in the spectroscopic sample are usually much brighter and have more photometric data than the other sources.

\subsection{Photometric Redshift Fitting}

We use the Zurich Extragalactic Bayesian Redshift Analyzer (ZEBRA; Feldmann et al. 2006) to compute the photo-z's. ZEBRA provides a maximum-likelihood estimate for the photo-z's of individual galaxies or AGNs, combining together some useful features such as automatic corrections of the SED templates to best represent the SEDs of real sources at different redshifts (the training procedure). Briefly, for every input SED template in each user-specified redshift bin, ZEBRA generates a new SED template that simultaneously minimizes the $\chi^{2}$ values for all the training sources (with spec-z's available) that use this input template as the bestfit template. The procedure is iterated several times, selecting the best-fit template from also the previous trained templates from the second iteration onward (see more details in Feldmann et al. 2006). The template correction/training process substantially reduces template mismatches and improves the photo-z accuracy. In the template-training mode, we used the spectroscopic sample as a training set to improve the SED templates.

To construct a suitable library of SED templates, we followed a procedure similar to that in Salvato et al. (2009), which calculated photo-z's for the XMM-Newton COSMOS sources. First, we ran ZEBRA on the spectroscopic sample of 220 sources, fixing the redshifts to their spec-z's and using the 259 PEGASE galaxy templates that cover different galaxy types (elliptical, spiral, and starburst) and span a wide range of star-formation history and intrinsic extinction (Grazian et al. 2006). Of these templates, 77 were used by the $220 \mathrm{spec}-$ troscopic sources. Second, we repeated the above procedure using the 10 AGN templates of Polletta et al. (2007) instead, which include a variety of empirical quasar and Seyfert templates. Eight out of the 10 AGN templates were used, including three type 1 and five type 2 AGNs. Third, to account for contributions from both the nuclear emission and host-galaxy emission in AGNs, we constructed a series of hybrid templates with the five most frequently used galaxy templates and four most frequently used AGN templates. For each pair of AGN and galaxy templates, the templates were normalized by the total 3000-4000 A fluxes, and nine hybrids with varying galaxy/AGN ratios (from 90:10 to 10:90) were created. This procedure produced a set of 265 templates that consists of 180 hybrid templates, 77 galaxy templates, and 8 AGN templates. We chose a much larger sample of SED templates than that in Salvato et al. (2009), which has only 30 templates, so that we are less affected by selection effects when estimating the photo-z quality using the spectroscopic sample (see $\$ 3.3$ below). We have tested several smaller SED libraries, which provide little improvement in the photo-z quality for the spectroscopic sample, whereas limited SED templates will probably hurt the performance of ZEBRA on the photometric sample as they might not represent the typical SEDs of the sources without spec-z's.

When using ZEBRA to fit the SEDs, we set most of the parameters to their default values. The filter responses and galaxy templates were resampled to a uniform wavelength mesh with a resolution of $4 \AA$ between $400 \AA$ and $10 \mu \mathrm{m}$. To minimize possible spurious boundary effects, we ran the template-correction mode twice with two different sets of redshift bins and then averaged the resulting improved tem- 

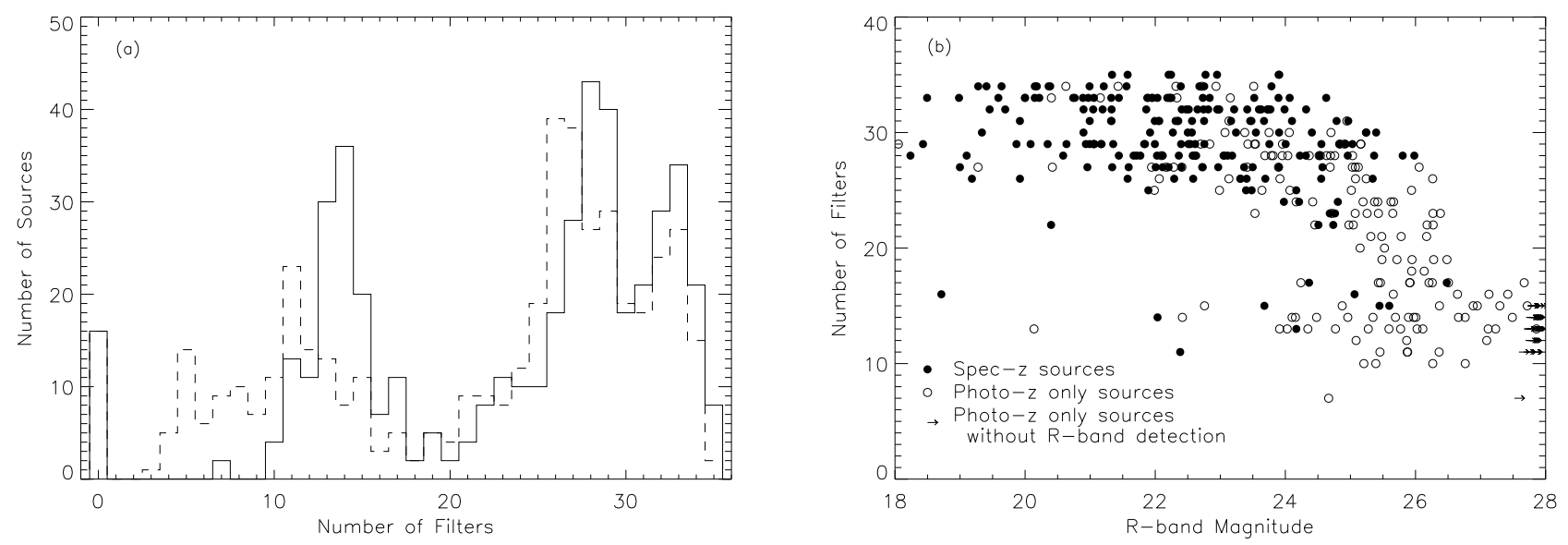

FIG. 8.- (a) Distributions of the number of filters used in the photo-z calculation (solid histogram) and the number of filters in which a source is detected (dashed histogram). The difference between these two histograms indicates the upper-limit information used in the calculation. The maximum number of filters used in the photo-z calculation is 35 , and the minimum number is 7 . The 16 sources without identifications have a filter number of 0 . The apparent bimodalities of the distributions are due to the inclusion or exclusion of the COMBO-17 data, which gives a difference of $\approx 17$ in the number of filters. (b) Number of filters used in the photo-z calculation vs. the WFI/MUSYC $R$-band magnitude for sources with spec-z's (filled dots) and sources without spec-z's (open dots). For 62 sources that do not have $R$-band data, their $R$-band upper limits are shown. Sources in the spectroscopic sample are typically much brighter and have more photometric data than the other sources. A few bright (e.g., $R<22$ ) sources have relatively small numbers of filters, because their COMBO-17 data are not available (not detected due to source blending). Also note that for very faint sources, e.g., $R>26$, most of the data available are upper limits.

plates to create a final SED library. For the input photometric catalog, we employed a minimum magnitude error of 0.06 for each filter, which effectively avoids cases where a few data points with unrealistically small errors have very large weights and thus drive the overall fitting results. We applied a luminosity prior of $M_{B} \leq-18$, which forces a lower limit on the absolute $B$-band magnitude. There are only two sources in the spectroscopic sample with fainter magnitudes. Applying such a prior leads to higher photometric redshifts for such faint sources, but tests show that it reduces the percentage of catastrophic photo-z failures (outliers in a spec-z vs. photo-z plot) considerably. ZEBRA does not take upper limits in the input data. To circumvent this problem, we used a "workaround" by treating the $1 \sigma$ upper limits as actual detections and setting the flux ranges to be from 0 to $2 \sigma$ (R. Feldmann 2009, private communication). These data points generally have a small weight in the SED fitting because of the large error bars, but they do help ZEBRA to determine the correct location of the Lyman break and thus give a better estimate of the photo-z, especially for sources with upper limits only in the NUV/FUV, and sources with detections only in the NIR/IR.

For each source, the ZEBRA SED fitting procedure generates a two-dimensional probability distribution in template and redshift space; the probabilities indicate the quality of fit based on the maximum-likelihood analysis. The best fit with the highest probability (corresponding to the lowest $\chi^{2}$ value) is chosen as the final photo-z and SED template for each source. In the photometric sample, $\approx 55 \%$ of the sources having multiple local maximum probabilities. As a supplement, we compare these local maximum probabilities, select the fit with the second highest probability (corresponding to the second lowest $\chi^{2}$ value), and derive an alternative photo-z, if the second highest probability is within two orders of magnitude of the highest value $(\approx 40 \%$ of the sources with multiple local maximum probabilities). If available, alternative photo-z's are provided for the photometric sample in Table 5 (see below).

Stars in the sample were identified by visual inspection of the GOODS-S and GEMS HST images and also the SED fitting results (the AGN/galaxy templates we used generally cannot fit a star SED and there is a strong optical excess). Every source was visually examined, though we focused on sources identified as stars by the COMBO-17 and GOODS-S MUSIC classifications, and sources with low fullband X-ray-to-z-band flux ratios. There are six stars identified, with X-ray source IDs 36, 172, 206, 352, 400, and 420; all of them have flux ratios $\left(F_{0.5-8.0 \mathrm{keV}} / F_{z}\right)$ smaller than 0.01 .

\subsection{Photometric Redshift Results}

Photo-z's were obtained for the 440 X-ray sources in the spectroscopic and photometric samples. They are listed in Table 5, with the details of the columns given below.

- Column (1): the X-ray source ID number.

- Column (2): the spec-z, set to "-1" if not available.

- Column (3): the photo-z derived using ZEBRA. Set to " 0 " for the six stars and " -1 " for the 16 sources without identifications.

- Columns (4) and (5): the $1 \sigma$ confidence interval (lower and upper bounds) of the photo-z. We caution that the error estimate is probably not be a good indicator of the accuracy of the photo-z (see $\$ 3.4$ for more details).

- Columns (6) and (7): the number of filters in which the source is detected, and the number of filters used in the photo-z calculation. The difference between these two numbers is the number of upper limits used; see Table 4 and Figure 8 for more details.

- Column (8): the reduced $\chi^{2}$ value for the best-fit result, $\chi_{\text {dof }}^{2}$, indicating the quality of fit. A value that deviates significantly from unity is generally caused by disagreement between the photometric data in different catalogs, which is often likely the result of AGN variability.

- Column (9): the alternative photo-z, set to "-1" if not available. There are 49 sources having an alternative photo-z. 
- Column (10): the reduced $\chi^{2}$ value for the alternative photo-Z, $\chi_{\text {dof,alt }}^{2}$.

- Column (11): a flag indicating whether the X-ray source is detected in the optical. A value of " 0 " means no optical detection, in which case the photo-z was calculated using only the IR/NIR data and the optical upper-limit information (and is probably not very reliable). There are 20 sources in the photometric sample that do not have optical detections.

- Column (12): Spec-z reference number; numbers 1 to 8 correspond to Le Fèvre et al. (2004), Szokoly et al. (2004), Zheng et al. (2004), Mignoli et al. (2005), Ravikumar et al. (2007), Vanzella et al. (2008), Popesso et al. (2009), and Silverman et al. (2010, in preparation), respectively.

The highest spec-z for a CDF-S X-ray source is 3.70. However, the derived photo-z's show that ten sources in the photometric sample have $z_{\text {photo }}=4.3-7.6$. These sources are generally not detected in the optical, or only have a faint optical counterpart. They are detected in 4-10 photometric bands, and the resulting photo-z's are probably not very reliable. In fact, eight of these objects have an alternative photo-z of 2.7-4.4 as listed in Table 5 (for comparison, only 41 of the remaining 210 sources in the photometric sample have an alternative photo-z). In Figure 9a, we show the SEDs along with the best and alternative fitting templates of the five sources with $z_{\text {photo }}>5$ (source 95 does not have an alternative photo-z). The SEDs and fitting templates for the five $z_{\text {photo }} \approx 4-5$ sources (XIDs 7, 21, 191, 404, and 439) are qualitatively similar to those shown in Figure 9 a. Given the limited SED data available, both the best and alternative fitting results appear acceptable. We estimated the intrinsic hydrogen column densities $\left(N_{\mathrm{H}}\right.$; see the end of this section for details of $N_{\mathrm{H}}$ estimation) for these sources. Two sources (XIDs 7 and 439) have estimated $N_{\mathrm{H}}$ values smaller than $6 \times 10^{22} \mathrm{~cm}^{-2}$, and another seven sources have $N_{\mathrm{H}}$ values between $1 \times 10^{23}$ and $8 \times 10^{23} \mathrm{~cm}^{-2}$. Source 261 has a very hard X-ray spectrum, which requires a Compton-thick column density $\left(N_{\mathrm{H}} \approx 1.9 \times 10^{24} \mathrm{~cm}^{-2}\right.$; an outlier compared to the $N_{\mathrm{H}}$ values of all the other X-ray sources) to produce the observed X-ray counts at $z_{\text {photo }}=5.2$, and is thus more likely located at its alternative redshift of $z_{\text {alt }}=2.7$, which only requires $N_{\mathrm{H}} \approx 8 \times 10^{23} \mathrm{~cm}^{-2}$. Given the properties above and the scarcity of $z>4$ AGNs in the Chandra deep fields, e.g., only three such objects are identified spectroscopically in the $\approx 2$ Ms CDF-N (e.g., Waddington et al. 1999; Vignali et al. 2002; Barger et al. 2003) ${ }^{32}$, we consider that at least half of the ten sources with high photo-z's are probably at intermediate redshifts and the alternative photo-z's are preferable for them. In the following analysis, we will still use the best-fit photo-z's for all the sources.

Rodighiero et al. (2007) estimated photo-z's for a sample of optically undetected objects, including three of our Xray sources (XIDs 289, 306, and 322, with photo-z's $\approx 4$, $\approx 4$, and $\approx 8$ ). Their estimated photo-z for source 306 is more consistent with its alternative photo-z in our catalog

\footnotetext{
32 One X-ray source (XID 618) in the Giacconi et al. (2002) catalog for the $\approx 1 \mathrm{Ms}$ CDF-S has a spec-z of 4.759 (Vanzella et al. 2006; Fontanot et al. 2007). The source was detected only by SEXTRACTOR in Giacconi et al. (2002), and was not detected in the Alexander et al. (2003) catalog for the $\approx 1 \mathrm{Ms}$ CDF-S or the L08 catalog for the $\approx 2 \mathrm{Ms}$ CDF-S.
}

(see Fig. 9a). We note that Mainieri et al. (2005) also derived a single solution for the photo-z of source 95 (see Fig. $9 \mathrm{a}$ ), though with a higher value, $z_{\text {photo }}=7.9$; this source may be a $z>5$ AGN candidate. There are also at least two $z>5$ AGN candidates among the unidentified X-ray sources (see \$4.11. Koekemoer et al. (2004) studied seven extreme X-rayto-optical ratio sources (EXOs) in the $\approx 1 \mathrm{Ms}$ CDF-S. These sources are all included in the L08 catalog, with source IDs of 95, 98, 133, 189, 235, 290, and 404. Sources 95 and 404 have photo-z's of 5.7 and 4.7 in our catalog, respectively, while the other five sources have photo-z's ranging from 2.2 to 3.2. It thus appears that most of the EXOs are at moderate redshifts (e.g., Mainieri et al. 2005).

The distribution of all the derived photo-z's is shown in Figure 10 $\mathrm{k}$. The median photo- $\mathrm{z}$ for all the sources is 1.3, and it is 2.0 for just the photometric sample. For comparison, the median spec-z is only 0.7 for the spectroscopic sample. ${ }^{33}$ Between $z=1$ and $z=4$, there are 179 sources in the photometric sample and only 84 sources in the spectroscopic sample. It is reasonable that sources without spectroscopic information are in general at higher redshifts. We calculated the rest-frame $0.5-8.0 \mathrm{keV}$ luminosities for the X-ray sources using the spec-z's and photo-z's. The intrinsic absorption was corrected for by assuming an intrinsic X-ray power-law photon index of $\Gamma=1.8$ and using XSPEC (Version 12.5.0; Arnaud 1996) to derive the appropriate intrinsic hydrogen column density $\left(N_{\mathrm{H}}\right)$ that produces the observed $\mathrm{X}$-ray band ratio (defined as the ratio of count rates between the hard and soft bands). A plot of luminosity versus redshift is shown in Figure 10p. Sources in the photometric sample have larger luminosities than spec-z sources on average. We selected AGNs from the X-ray sources following the criteria used by Bauer et al. (2004). Sources with $0.5-8.0 \mathrm{keV}$ $\mathrm{X}$-ray luminosities $L_{0.5-8.0 \mathrm{keV} \text {,rest }}>3 \times 10^{42} \mathrm{erg} \mathrm{s}^{-1}$, effective photon indices $\Gamma_{\text {eff }}<1$, or X-ray-to-optical ( $R$-band) flux ratios $F_{0.5-8.0 \mathrm{kev}} / F_{R}>0.1$ are considered to be AGNs. In this way, we classified 354 AGNs among the 440 identified X-ray sources, 150 in the spectroscopic sample and 204 in the photometric sample. It thus appears that the majority of the Xray sources in the photometric sample are AGNs at relatively high redshifts. In Figure $9 \mathrm{~b}$, we show the median SED of 330 AGNs with detections in at least six photometric bands and having redshifts $z<4$, along with the median SEDs in several different X-ray luminosity bins. The median SEDs in the optical are dominated by stellar light from the host galaxies, consistent with our SED fitting results (see §3.6).

\subsection{Photometric Redshift Accuracy}

To check the quality of the photo-z's, we compared the photo-z's to the spec-z's for the 220 sources in the spectroscopic sample (see Figures 11k, 12 $\mathrm{a}$, and 13 $\mathrm{a}$ ). The catastrophic failures (outliers) are defined as having $|\Delta z| /\left(1+z_{\text {spec }}\right)>0.15$, where $\Delta z=z_{\text {photo }}-z_{\text {spec }}$. There are 3 outliers $(1.4 \%)$ in the spectroscopic sample. The outliers typically still have acceptable SED fitting results, including small photo-z errors, normal reduced $\chi^{2}$ values, and no obvious alternative photo-z choices, and thus cannot be discriminated without the spec-z information. Specifically, there are two relatively bright outliers in Figure 13 a (sources 243 and 134 , with $R \approx 20.0$ and $R \approx 22.8$ ). One of them

33 This median spectroscopic redshift may be partially related to the two apparent redshift spikes at $z=0.67$ and 0.73 in the CDF-S (e.g., Gilli et al. 2003; Silverman et al. 2008). 

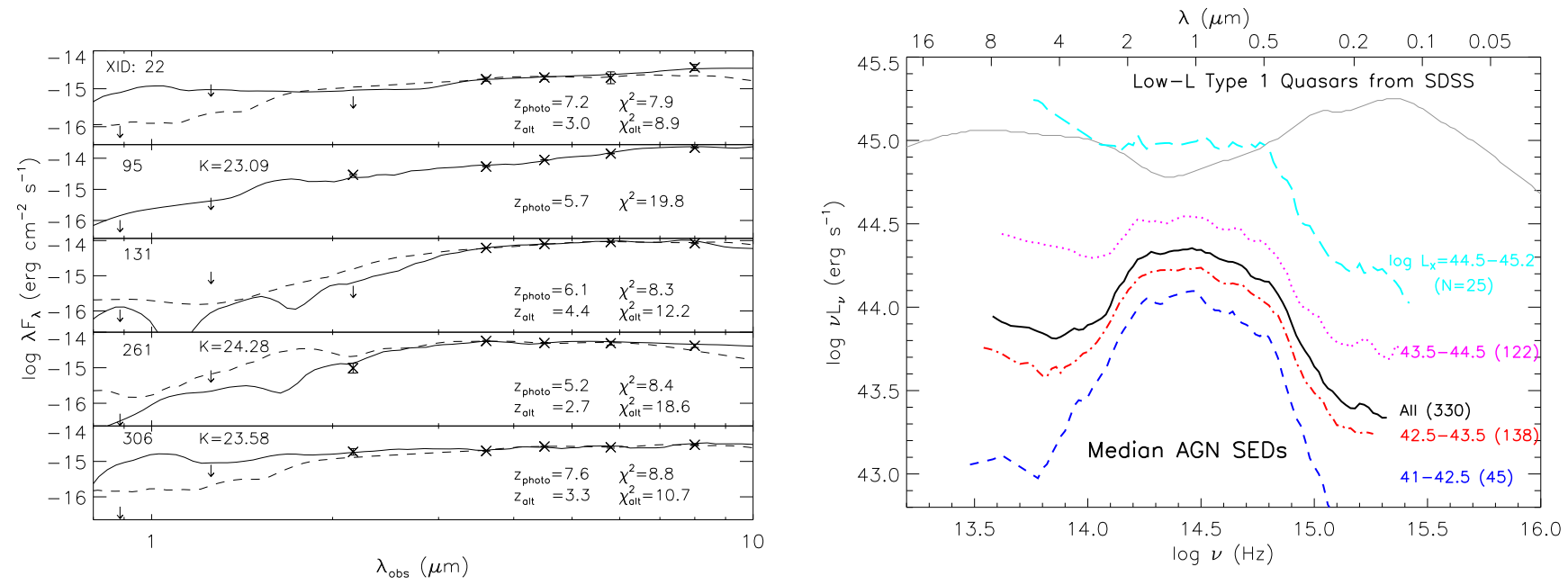

FIG. 9.- (a) SEDs and best-fit templates (solid curves) for the five X-ray sources with photo-z's greater than 5. Also shown are the alternative fitting templates (dashed curves) for four of the sources. These templates are either galaxy templates or hybrid AGN templates. None of the five sources is detected blueward of $K$ band (the $K$-band magnitude is indicated in each panel if available). The $1 \sigma$ upper limits on the $z, J$, and $K$ bands are shown if there are no detections. The $1 \sigma$ upper limits do not set tight constraints on the actual fluxes, so they may appear below the the SED templates in some cases. $(b)$ The median SEDs of $z=0-4$ AGNs in the CDF-S, derived from the photometric data and best-fit SED templates. The $24 \mu \mathrm{m}$ data from the Spitzer Far Infrared Deep Extragalactic Legacy Survey (FIDEL; Dickinson et al. 2010, in preparation) were also used. The cyan long-dashed, magenta dotted, black solid, red dash-dotted, and blue dashed curves represent median SEDs in different X-ray luminosity bins (as labeled, along with the number of AGNs contributing to each median SED). The grey solid curve represents the low-luminosity quasar mean SED from Richards et al. (2006) with the host-galaxy contribution subtracted. The SEDs of CDF-S AGNs are generally dominated by host-galaxy light and show heavy extinction in the UV. [See the electronic edition of the Journal for a color version of this figure.]
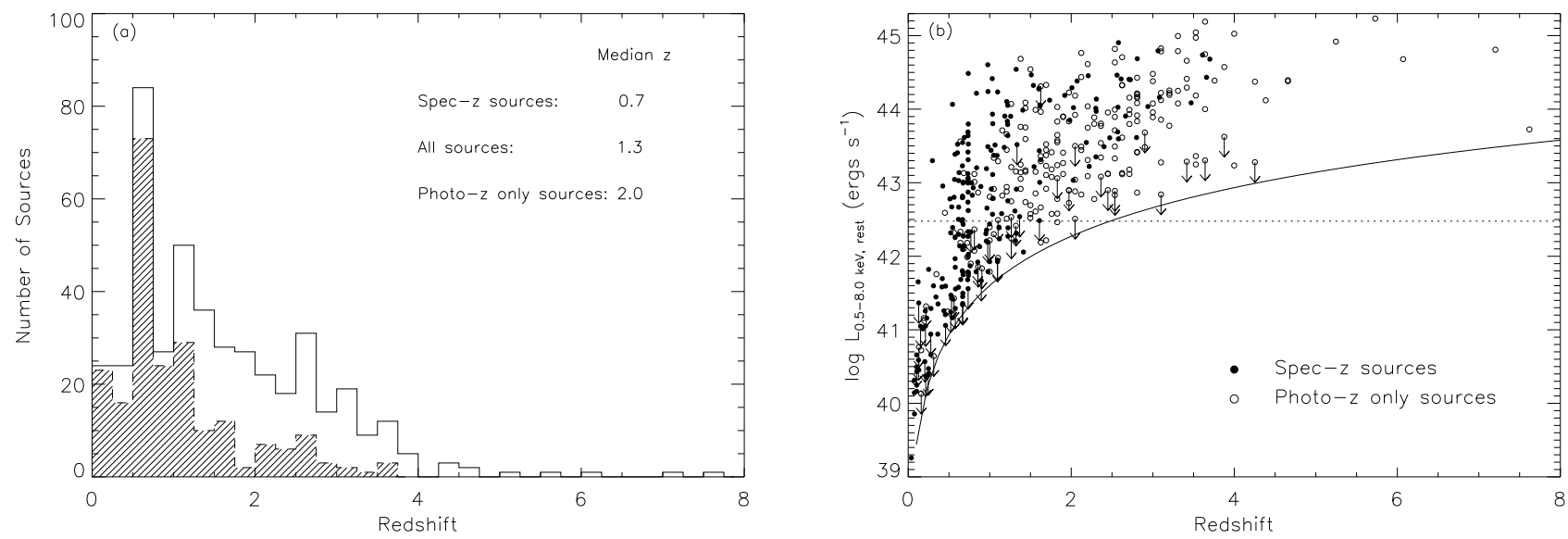

FIG. 10.- (a) Histogram showing the distribution of photo-z's. The shaded region shows the distribution of spec-z's. Sources with only photo-z's are generally at higher redshifts than spec-z sources. (b) Redshift-luminosity distribution for spec-z sources (filled dots) and photo-z sources (open dots). The solid curve represents the limiting $0.5-8.0 \mathrm{keV}$ luminosity at the center of the CDF-S region (L08), assuming $\Gamma=1.8$ and $N_{\mathrm{H}}=10^{22} \mathrm{~cm}^{-2}$. The dotted line indicates $L_{0.5-8.0 \mathrm{keV}, \text { rest }}=3 \times 10^{42} \mathrm{erg} \mathrm{s}^{-1}$. Some of the photo-z's for the ten objects with $z_{\text {photo }}>4$ are probably not reliable.

(source 243) is caused by the bright GALEX FUV detection $\left(m_{\mathrm{FUV}}=21.1\right)$, which should have been suppressed due to the Lyman break for a source at $z_{\text {spec }}=1.03$; the FUV flux may be contributed by a few surrounding faint sources. The other one is produced by our luminosity prior of $M_{B} \leq-18$, which pushes the photo-z to a larger incorrect value $\left(z_{\text {spec }}=0.12\right.$ and $\left.z_{\text {photo }}=0.64\right)$.

We also use two other indicators to evaluate the photo- $z$ quality, the normalized median absolute deviation of $\Delta z$ $\left(\sigma_{\mathrm{NMAD}}\right)$ and the average absolute scatter $(A A S)$, which are defined as

$$
\sigma_{\mathrm{NMAD}}=1.48 \times \operatorname{median}\left(\frac{|\Delta z-\operatorname{median}(\Delta z)|}{1+z_{\mathrm{spec}}}\right),
$$

and

$$
A A S=\operatorname{mean}\left(\frac{|\Delta z|}{1+z_{\text {spec }}}\right)
$$

$\sigma_{\mathrm{NMAD}}$ is a robust indicator of the photo-z accuracy after the exclusion of outliers (e.g., Maronna et al. 2006; Brammer et al. 2008), while $A A S$ shows the mean scatter including the contribution from outliers. For our spectroscopic sample, $\sigma_{\mathrm{NMAD}}=0.010$ and $A A S=0.013$. These parameters, along with Figure $11 \mathrm{a}$, indicate that the photo-z's for the spectroscopic sample are very reliable in terms of both the outlier percentage and accuracy. We show in Figure 1 the SED fitting result for source 312 as an example; the derived photo-z for this source is accurate to within $1 \%\left[|\Delta z| /\left(1+z_{\text {spec }}\right)=0.005\right]$.

However, the above evaluation does not represent the quality of the photo-z's for sources in the photometric sample, because the SED templates were corrected using the spec-z information, and we are thus biased to get optimal fitting results for sources with spec-z's. Therefore, we performed a "blind test" to derive a more realistic estimate of the overall photo-z accuracy. We randomly picked $3 / 4$ of the sources 
in the spectroscopic sample, and only the spec-z's of these sources were used to train the SED templates. The photo-z's of the other $1 / 4$ of the sources are calculated based on the improved templates, and these are the blind-test sources as there was no prior knowledge utilized about their spec-z's. The above process was repeated 20 times to create a statistically significant sample including 1110 blind-test sources (the blind-test sample). Applying the same quality indicators above to the blind-test sample, we get an outlier percentage of $8.6 \%, \sigma_{\mathrm{NMAD}}=0.059$, and $A A S=0.065$. The results are shown in Figures $11 \mathrm{~b}, 12 \mathrm{~b}$, and $13 \mathrm{~b}$. The photo- $\mathrm{z}$ accuracy does not appear to degrade at high redshifts $(z \approx 2-4)$, and the accuracy declines at faint $R$-band magnitudes (the outlier fractions for the $R>22.5$ and $R \leq 22.5$ samples are $11.4 \%$ and $6.1 \%$, respectively). Note that among the 1110 sources in the blind-test sample, some have the same spec-z, photo-z, and $R$ magnitude (same source in the spectroscopic sample, and different training samples do not alter the best-fit photo-z), and thus cannot be distinguished in Figures $11 \mathrm{~b}$ and $13 \mathrm{~b}$ (i.e., they appear as the same data point). The effective size of the blind-test sample should be around 220, as we only have this number of sources in the spectroscopic sample.

In Figures 14 and $14 \mathrm{~b}$, we show the distributions of the $1 \sigma$ photo-z errors estimated from the ZEBRA SED fitting for the 440 X-ray sources (see Table 5) and the blind-test sample, respectively. The spectroscopic sources generally have smaller photo-z errors than the photometric sources, mainly because of their better multiwavelength coverage; the ZEBRA photo-z errors also appear to decrease toward brighter magnitudes, for the same reason. Sources with an alternative photo-z (blue squares in Fig. 14a) tend to have a large photo-Z error, and the outliers (red stars in Figs. 14 and 14b) cannot be distinguished based on their photo-Z errors. The ZEBRA photo-z errors were calculated in the two-dimensional redshift-template space. However, the template plays a minor role in the error calculation, unless there are multiple competitive SED fits with very close $\chi^{2}$ values. Therefore, the errors for most sources were determined using essentially only the best-fit template, and are generally underestimates of the real errors. We compared the ZEBRA $1 \sigma$ photo-z errors to the real errors $(|\Delta z|)$ using the spectroscopic and blind-test samples, and the results are shown in Figure 14k. The ZEBRA photo-z errors only account for $\approx 30 \%$ and $\approx 17 \%$ of the real errors on average for the spectroscopic and blind-test samples, respectively. Similar behavior has been discovered for the photo-z errors derived using other photo-z codes (e.g., Hildebrandt et al. 2008), in the sense that the photo-z accuracy is not tightly correlated with the error estimate. A more realistic estimate of the photo-z errors for our sources is given by the $\sigma_{\text {NMAD }}$ parameter, about $1 \%$ for the spectroscopic sample and $6 \%$ for the blind-test sample.

Although we give an unbiased estimate of the photo-z quality using the blind-test sample, we note that there are three factors that could affect the results: (1) In the blind-test sample, only $3 / 4$ of the spec-z sources are used to train the templates, while in the final results, all the spec-z sources are used in the training. Thus the photo-z quality should be somewhat better due to the larger training sample. (2) The quality estimate is solely based on the spectroscopic sample, in which sources are generally brighter and have better photometric sampling than the sources in the photometric sample (see Fig. 8b). The photo-z quality for the latter might not be as good as what we derived here. Indeed, Hildebrandt et al. (2008) have shown that the outlier fractions for the latter sam- ples are typically larger by at least a factor of two. We performed another blind test, choosing the 182 spec-z sources with WFI $R<24$ as the training sample, and then calculating the photo-z's of the other 38 spec-z sources with $R \approx 24-26$. The resulting outlier fraction is $10.5 \%$ and $\sigma_{\mathrm{NMAD}}=0.067,{ }^{34}$ which are not greatly increased relative to the $8.6 \%$ outlier fraction and $\sigma_{\text {NMAD }}=0.059$ for the blind-test sample above. Note that these 38 faint spec-z sources are still significantly brighter than the sources in the photometric sample on average (see, e.g., Figure $8 \mathrm{~b}$ ); therefore an outlier fraction of $\approx 15-25 \%$ is probable for the faint sources $(R \gtrsim 26)$ in the photometric sample. (3) The spec-z's were used when we selected the SED templates. This should not affect the blind test significantly, as we chose a large and relatively complete SED library.

In summary, among the 220 photo-z's in the photometric sample, we expect there are $\approx 9 \%$ outliers for the $\approx 110$ relatively brighter sources $(R \lesssim 26)$, and the outlier fraction will rise to $\approx 15-25 \%$ for the fainter sources $(R \gtrsim 26)$. The typical photo-z accuracy is $\approx 6-7 \%\left(\sigma_{\mathrm{NMAD}}\right)$. The outlier fraction and photo-z accuracy do not appear to have a redshift dependence (for $z \approx 0-4$ ). Among the faint $R \gtrsim 26$ sources, 20 objects do not have any optical counterparts (see Table 5, including the five $z_{\text {photo }}>5$ sources shown in Fig. 9a), and the photo-z's were calculated using only the IR/NIR photometric data and the optical upper-limit information. Caution should be taken when using these photo-z's. Moreover, the alternative photo-z's provided for the 49 sources may occasionally be a better choice than the best-fit photo-z's (e.g., for some of the $z_{\text {photo }}>4$ sources).

Recently, the GOODS/VIMOS spectroscopic campaign has provided two additional secure spec-z's for our X-ray sources (Balestra et al. 2010), $z_{\mathrm{spec}}=0.740$ for source 175 and $z_{\mathrm{spec}}=$ 2.586 for source 372 . Their photo-z's in our catalog are 0.56 and 2.71, with accuracies of $|\Delta z| /\left(1+z_{\text {spec }}\right) \approx 10 \%$ and $3.6 \%$, respectively.

\subsection{Comparison with Other AGN Photometric-Redshift Accuracies}

The photo-z quality of the spectroscopic sample $(1.4 \%$ outlier and $\left.\sigma_{\mathrm{NMAD}}=0.01\right)$ is comparable to those of recent photo-z studies for the COSMOS optical (e.g., Ilbert et al. 2009) and X-ray (Salvato et al. 2009) sources. Compared to the blind-test result (46 sources) for the COSMOS X-ray sources (Salvato et al. 2009) with $10.9 \%$ outliers and $\sigma_{\text {NMAD }} \approx 0.023$, our result has a slighter smaller fraction $(8.6 \%)$ of outliers but a larger $\sigma_{\mathrm{NMAD}}(0.059)$. Note that the CDF-S and COSMOS X-ray sources are two different populations. The $\approx 2 \mathrm{Ms} C \mathrm{CDF}-\mathrm{S}$ sources have an average Chandra effective exposure of $\approx 1 \mathrm{Ms}$, while the COSMOS sources have an average effective $X M M-N e w t o n$ exposure of $\approx 50 \mathrm{ks}$. In our spectroscopic sample, $\approx 38 \%$ of the sources have $H S T$ $i>22.5$, and in the COSMOS spectroscopic sample, only $\approx 10 \%$ of the sources have Canada-France-Hawaii Telescope $i^{*}>22.5$ (Salvato et al. 2009). Our photo-z quality is at least comparable to that for the COSMOS X-ray sources, considering that we are dealing with a fainter and thus more challenging sample. One advantage in the photo-z work for the COSMOS X-ray sources is that a correction was made for optical

\footnotetext{
34 The photo-z quality does not appear to degrade from $R>22.5$ to $R>24$ (see Figure 13b), maybe due to the small sample size of $R>24$ sources. Therefore the outlier fraction here is comparable to that $(11.4 \%)$ for the $R>$ 22.5 sources in the blind-test sample above.
} 

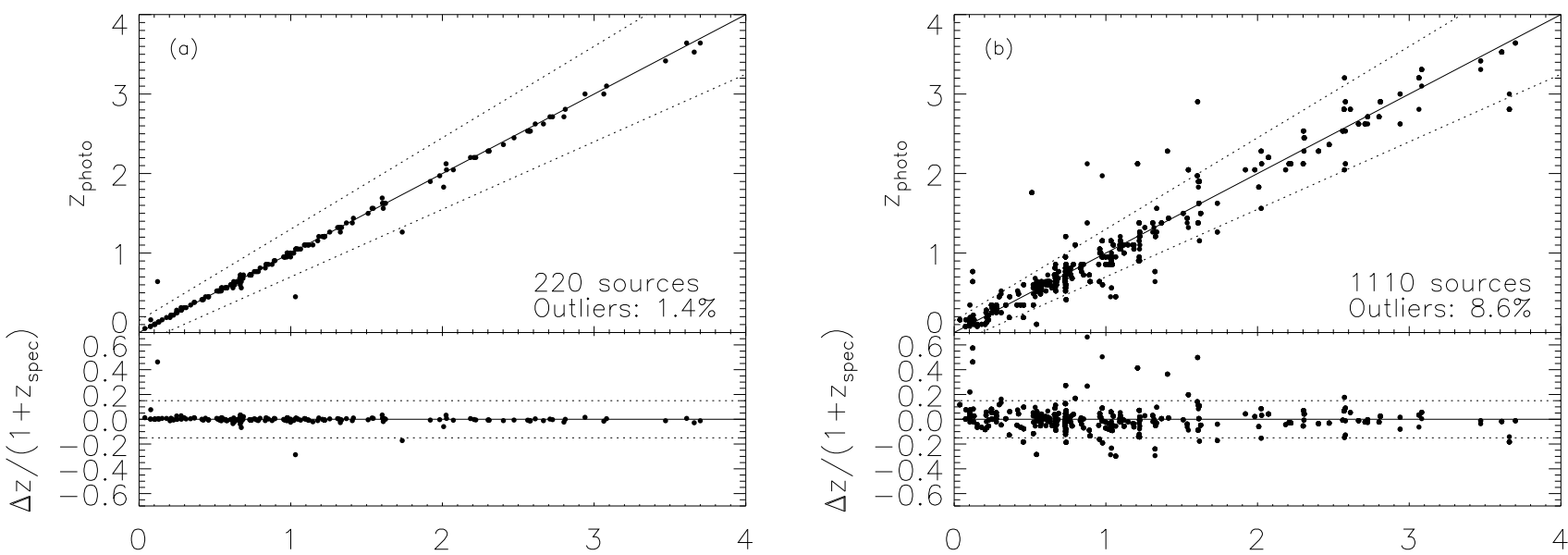

FIG. 11.- Comparison of the photo-z's Zand spec-z's for $(a)$ the spectroscopic sample and $(b)$ the blind-test sample. Thes apper panels show the distribution of $z_{\text {spec }}$ vs. $z_{\text {photo }}$, and the lower panels show $z_{\text {spec }}$ vs. $\Delta z /\left(1+z_{\text {spec }}\right)$. The solid lines indicate $z_{\text {photo }}=z_{\text {spec }}$, and the dotted lines represent relations of $z_{\text {photo }}=$ $z_{\text {spec }} \pm 0.15\left(1+z_{\text {spec }}\right)$, which define the outlier limits. Note that one data point can represent several sources in the blind-test sample $(b)$. The photo-z's and spec-z's agree very well for the spectroscopic sample, while the photo-z's and spec-z's differences are considerably larger for the blind-test sample. The photo-z accuracy does not appear to degrade at high redshifts $(z \approx 2-4)$.
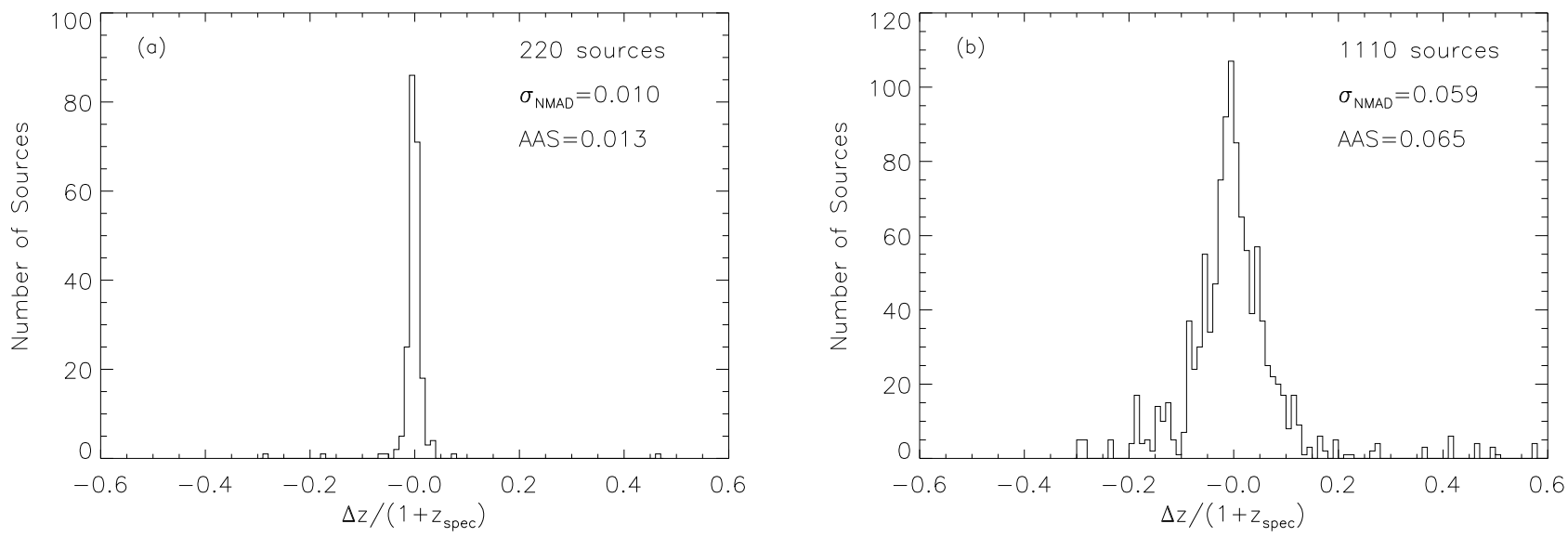

FIG. 12.- The distribution of the photo-z accuracy, $\Delta z /\left(1+z_{\text {spec }}\right)$, for $(a)$ the spectroscopic sample and $(b)$ the blind-test sample.
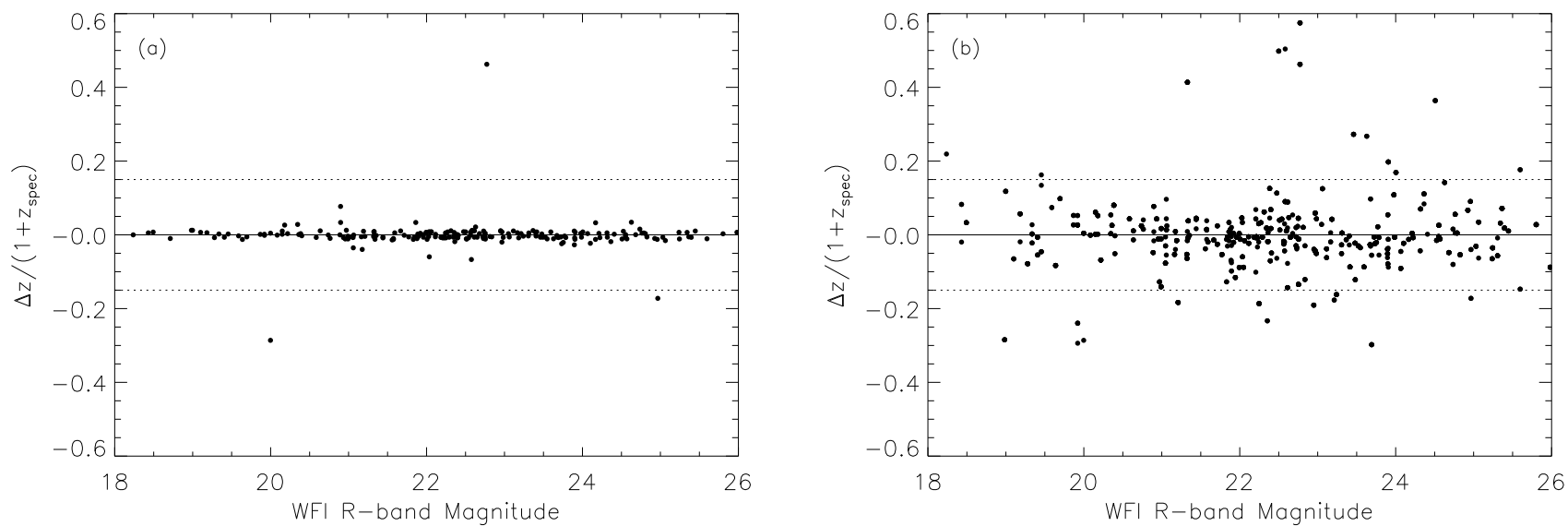

FIG. 13. - The photo-z accuracy, $\Delta z /\left(1+z_{\mathrm{spec}}\right)$, vs. WFI $R$-band magnitude for $(a)$ the spectroscopic sample and $(b)$ the blind-test sample. The solid lines indicate $z_{\text {photo }}=z_{\text {spec }}$, and the dotted lines represent relations of $z_{\text {photo }}=z_{\text {spec }} \pm 0.15\left(1+z_{\text {spec }}\right)$. Note that one data point can represent several sources in the blind-test sample $(b)$. The photo-z accuracy declines at faint $R$-band magnitudes. 

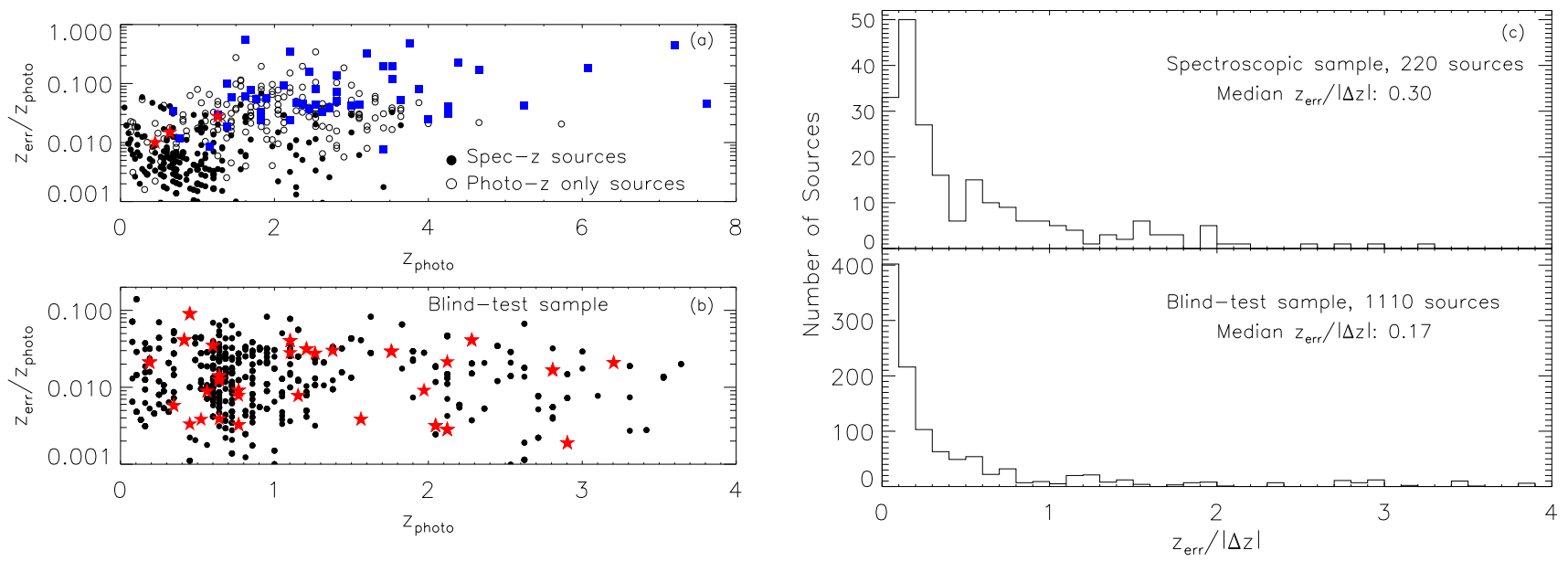

FIG. 14. - The distributions of the photo-z errors derived from the ZEBRA SED fitting for (a) the spectroscopic (filled dots) and photometric (open dots) samples and $(b)$ the blind-test sample. The ZEBRA photo-Z error is calculated as $z_{\text {err }}=\left(z_{\text {upper }}-z_{\text {lower }}\right) / 2$. The red stars represent outliers in the spectroscopic and blind-test samples. The blue squares represent sources with an alternative photo-z. In (c), we show the distribution of the ZEBRA photo-z errors in units of the real photo-z errors $(|\Delta z|)$ for the spectroscopic sample and the blind-test sample. The ZEBRA photo-z errors generally underestimate the real errors by a factor of $\approx 3-6$. [See the electronic edition of the Journal for a color version of this figure.] 
AGN variability, which helps to reduce the outlier fraction and $\sigma_{\text {NMAD }}$ (Salvato et al. 2009). Given the nature of the photometric data in the CDF-S, i.e., multiwavelength observations are not separated in well-defined epochs, source variability is difficult to take into account. However, we also note that for the moderate-luminosity AGNs in our sample, the SEDs are often dominated by host-galaxy light (see Fig. 9b), and thus AGN variability may play a smaller role for our sources than for the brighter COSMOS AGNs.

Compared to the photo-z quality for the $342 \mathrm{X}$-ray sources in the $\approx 1 \mathrm{Ms}$ CDF-S (Zheng et al.2004), with $\approx 15 \%$ outliers and $\sigma_{\text {NMAD }} \approx 0.08-0.1,35$ or the photo-Z quality of the 429 $\mathrm{X}$-ray sources in the $\approx 2 \mathrm{Ms}$ CDF-N (Barger et al. 2003), with $94 \%$ of the photo-z's accurate to within $\approx 25 \%$, our photo-Z accuracy estimated based upon the blind-test sample is significantly $(\gtrsim 50 \%)$ improved.

\subsection{AGN Classification and Best-Fit SED Templates}

The intrinsic hydrogen column density $N_{\mathrm{H}}$ estimated in $\$ 3.3$ (assuming an underlying $\Gamma=1.8$ ) can provide a basic AGN classification from the X-ray point of view, i.e., "absorbed" with $N_{\mathrm{H}} \geq 10^{22} \mathrm{~cm}^{-2}$ or "unabsorbed" with $N_{\mathrm{H}}<10^{22} \mathrm{~cm}^{-2}$. This method has limited diagnostic utility because of the complexity of AGN X-ray spectra. However, it suggests that $\approx 256(\approx 72 \%)$ of the 354 AGNs in the spectroscopic and photometric samples are absorbed, which is in general agreement with the $\approx 60-75 \%$ absorbed AGN fraction in the $\approx 1$ Ms CDF-S and $\approx 2$ Ms CDF-N based on X-ray spectral analysis (e.g., Bauer et al.|2004; Tozzi et al. 2006).

We investigated the best-fit SED templates selected for the 440 sources; about $98 \%$ of them are improved templates from the template-training procedure, indicating the importance of the training step. Of the 265 input templates (including their trained varieties), 105 are selected as the best-fit SED template for multiple sources and 107 are never used; the most frequently used template (a starburst-galaxy template) has been chosen for 22 sources. The best-fit SED templates can also provide a basic SED-based classification of the X-ray sources. Of the 440 source SEDs, 212 are best fit by galaxy templates, and the others are fit by AGN or hybrid templates, including 84 type 1 AGNs and 144 type 2 AGNs. For sources that are detected in only a few or none of the optical bands, this classification may be highly uncertain.

AGNs identified in the X-ray do not completely agree with the SED fitting results, as expected based upon disagreements between X-ray based and optical-spectroscopy based classification schemes (e.g., Matt 2002; Trouille et al. 2009). For example, of the 303 AGNs with detections in at least ten UV-to-IR bands, $125(41.3 \%)$ are best fit by galaxy templates. Despite the uncertainties in the SED fitting and the calculation of X-ray luminosities, some of these sources are likely the X-ray bright optically normal galaxies (XBONGs; e.g., Comastri et al. 2002), which have high X-ray luminosities but do not appear as AGNs in the optical. The nature of XBONGs remains somewhat mysterious: the properties of XBONGs are usually explained by heavy obscuration covering a large solid angle (e.g., Matt 2002; Rigby et al. 2006; Civano et al. 2007), dilution by host galaxies (e.g., Moran et al. 2002; Severgnini et al. 2003; Trump et al. 2009;

\footnotetext{
35 We also checked the photo-z quality of the Zheng et al. (2004) sources using 31 spec-z sources that did not have spec-z information in Zheng et al. (2004). The resulting outlier fraction is $\approx 26 \%$, and the outlier-excluded accuracy is $\sigma_{\mathrm{NMAD}} \approx 0.08$.
}

see also Fig. 9b), or a radiatively inefficient accretion flow (RIAF; e.g., Yuan \& Narayan 2004; Trump et al. 2009).

An apparent inconsistency also exists between the X-ray (unabsorbed versus absorbed) and SED-based (type 1 versus non-type 1, including type 2 and galaxies) classification schemes. Figure 15 displays the X-ray band ratio versus redshift for the 354 AGNs. The SED-based classification is color coded, while the X-ray classification is indicated by the theoretical band-ratio-redshift track. We study the classifications in two X-ray luminosity bins, divided by our median $\mathrm{X}$-ray luminosity, $L_{0.5-8.0 \mathrm{keV} \text {,rest }} \approx 2.6 \times 10^{43} \mathrm{erg} \mathrm{s}^{-1}$. We consider only the 303 AGNs with detections in at least ten UV-to-IR bands. In the low-luminosity (high-luminosity) bin, $42 \%(55 \%)$ of the type 1 AGNs are unabsorbed, $64 \%$ $(86 \%)$ of the type 2 AGNs are absorbed, and $71 \%(87 \%)$ of the AGNs fit by galaxy templates are absorbed. For relatively luminous X-ray sources, the two classification schemes tend to agree better, probably due to less fractional contamination from host-galaxy light. ${ }^{36}$ It appears that most of the non-type 1 sources ( $\approx 86 \%$ for luminous $X$-ray sources) are absorbed, while only about half of the type 1 sources are unabsorbed. The comparison between the X-ray and opticalspectroscopy classification schemes in Trouille et al. (2009) shows that $\approx 80 \%$ of the broad-line AGNs are unabsorbed and $\approx 67 \%$ of the non-broad-line AGNs are absorbed. Therefore, the X-ray versus optical-spectroscopy classification schemes agree better for unabsorbed/type $1 \mathrm{AGNs}$, compared to the Xray versus SED classification schemes. Note that the uncertainties in the simple X-ray classification scheme above are plausibly responsible for some of the classification discrepancy; detailed X-ray spectral analysis will provide a better $\mathrm{X}$-ray classification and give more insight into the problem of $\mathrm{X}$-ray versus SED classification inconsistency.

\section{DISCUSSION}

\subsection{Nature of the Unidentified X-ray Sources}

There are 16 unidentified sources, eight of which are probably related to a star (one source), galaxy groups and clusters (four sources), off-nuclear sources (two sources), or any of a number of galaxies at the edge of the CDF-S field (one source; see Col. 13 of Table 2). For the other eight X-ray sources (flagged with "NoID" in Col. 13 of Table 2, see Figure 4 for their locations), six were detected only at a falsepositive threshold of $1 \times 10^{-6}$, and two (sources 49 and 345) at $1 \times 10^{-8}$. At a threshold of $1 \times 10^{-8}$, we expect less than 0.2 false detections (see $\$ 3.2$ of L08). We visually checked the X-ray images of sources 49 and 345 and confirmed their apparent validity. We also investigated the Chandra events for these two sources and excluded the possibility of contamination from short-lived cosmic-ray afterglows. Therefore sources 49 and 345 are likely genuine X-ray sources. Source 49 is outside of the GOODS-S region. It was detected in all three X-ray bands $(\approx 117$ full-band counts) and was also present in the $\approx 1$ Ms CDF-S (Giacconi et al. 2002; Alexander et al. 2003). It is a hard X-ray source with a band ratio of 1.5 (corresponding to an intrinsic column density of $N_{\mathrm{H}} \approx 6 \times 10^{23} \mathrm{~cm}^{-2}$ assuming $z=5$ and $\Gamma=1.8$ ). Its $0.7-6 \mathrm{keV}$ $\mathrm{X}$-ray spectrum shows no obvious emission-line features. The X-ray-to-optical ( $z$-band) flux ratio is $F_{0.5-8.0 \mathrm{keV}} / F_{z} \gtrsim 50$.

\footnotetext{
36 Salvato et al. (2009) also made a similar comparison of the SED versus $\mathrm{X}$-ray classifications for the COSMOS X-ray sources, and found a generally better agreement $(\approx 80 \%)$, probably due to the fact that COSMOS samples brighter X-ray fluxes than the CDF-S.
} 

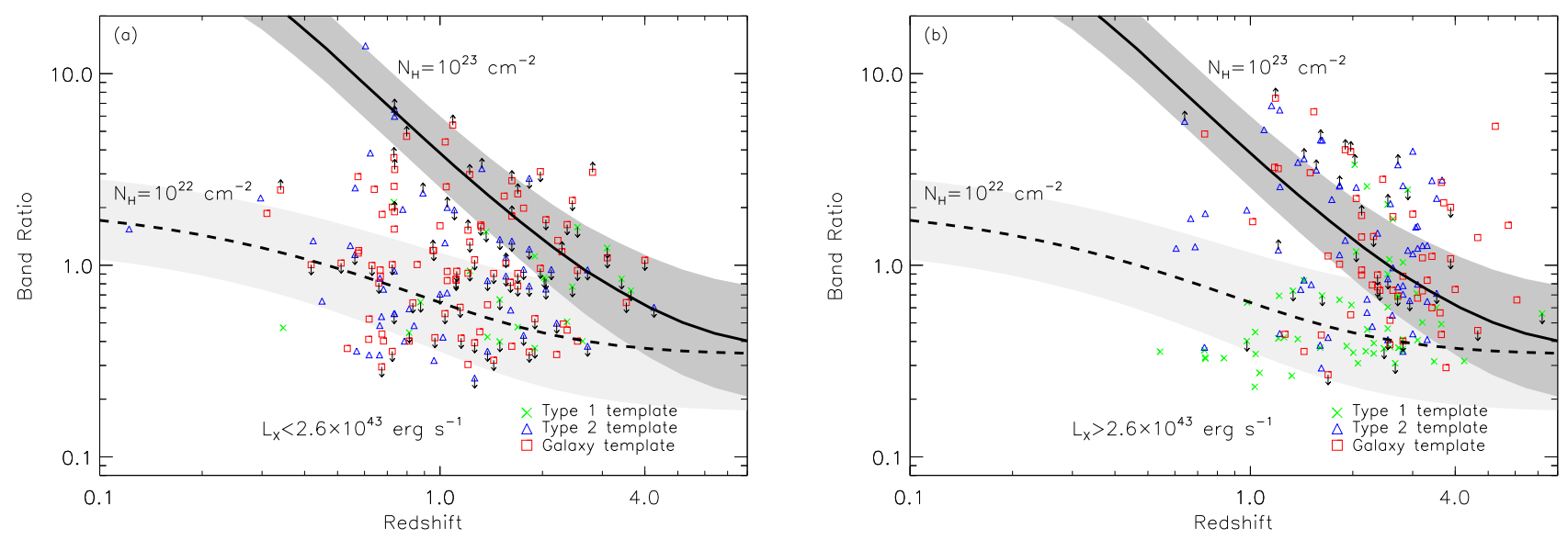

FIG. 15. - X-ray band ratio vs. redshift for the $(a)$ fainter and $(b)$ brighter halves of AGNs, separated by the median X-ray luminosity, $L_{0.5-8.0} \mathrm{keV}$, rest $=$ $2.6 \times 10^{43} \mathrm{erg} \mathrm{s}^{-1}$. Band ratios are defined as the ratio of count rates between the hard and soft bands. The green crosses, blue triangles, and red squares are sources whose SEDs are best-fit by type 1, type 2, and galaxy templates, respectively. Upper limits (lower limits) on the band ratios are shown when the sources are not detected in the hard band (soft band). The errors on the band ratios are not shown for clarity; the median error is $\approx 0.16$. The solid (dashed) curve and the dark (light) shaded region show the expected band ratios for AGNs with power-law photon indices $\Gamma=1.8 \pm 0.5$ and intrinsic absorption $N_{\mathrm{H}}=10^{23} \mathrm{~cm}^{-2}$ $\left(N_{\mathrm{H}}=10^{22} \mathrm{~cm}^{-2}\right)$. The X-ray (unabsorbed versus absorbed) and SED-based (type 1 versus non-type 1) classification schemes agree better for luminous AGNs; $\approx 86 \%$ of the non-type 1 sources are absorbed, and about half of the type 1 sources are unabsorbed. [See the electronic edition of the Journal for a color version of this figure.]

There appears to be marginal 3.6, 4.5, and $8.0 \mu \mathrm{m}$ emission from the X-ray position of source 49 . However, there is a relatively bright $[m(3.6 \mu \mathrm{m})=21.2]$ IR source nearby $\left(2.3^{\prime \prime}\right.$ away); even if the emission is from source 49 , it is completely blended with the bright source. There is also a faint VIMOS $U$-band source $(U=28.70 \pm 0.22) 1.3^{\prime \prime}$ away from source 49, which is not detected in any of the other bands. It was not considered a counterpart by the likelihood-ratio matching. Source 345 is inside the GOODS-S region and is only detected in the soft band with $\approx 6$ counts. The detection is likely significant because there are five counts landing on a single pixel. Its $\mathrm{X}$-ray-to-optical flux ratio is $F_{0.5-8.0 \mathrm{keV}} / F_{z} \gtrsim 10$. The nearest ONIR source to source 345 is $\approx 2.0^{\prime \prime}$ away.

The properties of sources 49 and 345 are comparable to the EXOs in Koekemoer et al. (2004), except that they do not have any clear detections in the IR or NIR bands (which EXOs generally have) and source 345 is relatively X-ray weak. Most of the EXOs are suggested to be dusty galaxies at moderate redshifts $(z \approx 2-3)$, and a small fraction could be AGNs at very high redshifts $(z>5$; e.g., Koekemoer et al. 2004; Mainieri et al. 2005). Given the apparent non-detection of these two sources in the deep IR and NIR images, they are unlikely to be dusty galaxies at moderate redshifts (i.e., their host galaxies should be detected). Therefore, sources 49 and 345 are more likely to be AGNs at very high redshifts that appear blank in all the current optical-to-IR catalogs due to finite-sensitivity effects. Using the luminosities and specz's or photo-z's of our X-ray sources, and the depths of the optical-to-IR catalogs, we estimate that $\approx 45(\approx 10 \%)$ of the sources would appear blank in all these bands if placed at $z=5$, and $\approx 10$ of them would still be detected in the Xray if at $z=5$. There is also the possibility that source 345 is an isolated neutron star, such as the so-called "Dim Isolated Neutron Stars" (e.g., Kaplan 2008), which emit thermal radiation with temperatures of $\approx 0.1 \mathrm{keV}$. This is not likely the case for source 49 because of its hard X-ray emission. Isolated neutron stars with faint X-ray fluxes such as source 345 would appear blank at optical-to-IR wavelengths, because of the high $\left(\approx 10^{3}-10^{4}\right)$ X-ray-to-optical flux ratios of these sources. Given the X-ray sensitivity of the CDF-S (L08) and the number counts of isolated neutron stars (e.g., Popov et al. 2000,2010 ), we expect $\approx 0.2$ detectable isolated neutron stars in the CDF-S field. Very deep NIR imaging or spectroscopic observations will help elucidate the nature of sources 49 and 345.

The six sources (XIDs 93, 106, 210, 280, 344, and 353) detected at a low-significance level of $1 \times 10^{-6}$ and without identifications could be spurious X-ray detections. Visual inspection indicates that most of these sources appear only marginally above the background. At a detection threshold of $1 \times 10^{-6}$, we conservatively estimated that there are $\approx 18$ false detections (see $\$ 3.2$ of L08), and the true number is likely $\approx 2-3$ times less (Alexander et al. 2003). Spurious X-ray detections could also exist in the identified source sample because of chance correlations. We estimate that the average possibility of matching a randomly placed $1 \times 10^{-6} \mathrm{X}$-ray source to an ONIR source is $\approx 11 \%$, using the same bootstrapping technique described in $\$ 2.3{ }^{37}$ If the six unidentified $\mathrm{X}$-ray sources are indeed spurious, we expect less than one $[6 \times 11 \% /(1-11 \%)]$ spurious $X$-ray source in our identified source sample. Therefore, there are up to $\approx 7$ spurious $X$-ray detections, six unidentified and one falsely identified. This number is roughly consistent with the expected value $(\approx 2-3$ times less than 18). If any of these six low-significance and unidentified sources is a real X-ray detection, it could have a similar nature as sources 49 and 345 .

\subsection{Future Prospects for Improved Source Identification}

Besides the unidentified X-ray sources, uncertainties are still present for the 442 identified sources (excluding the four additional likely cases), as there is $\mathrm{a} \approx 6.2 \%$ false-match probability and there are 21 primary counterparts from the MUSYC or SIMPLE catalogs with relatively less-accurate

${ }^{37}$ Essentially, this is an estimate of the false-match probability for X-ray sources detected at only the $1 \times 10^{-6}$ significance level. The false-match probability is higher than that for the entire X-ray sample $(\approx 6.2 \%)$, mainly because the positional uncertainties of these X-ray sources are relatively larger. 
$\left(0.2-0.3^{\prime \prime}\right)$ positions. In order to characterize the nature of all the X-ray sources in the $\approx 2 \mathrm{Ms} \mathrm{CDF}-\mathrm{S}$, efforts should focus on obtaining $(a)$ better positions for the counterparts and (b) a lower overall false-match probability. Better counterpart positions generally require deeper optical or NIR observations. Comparing the current deepest optical (GOODS-S $z$-band) and NIR (MUSIC $K$-band) catalogs, we consider the NIR band to be probably the most beneficial to push deeper, because of its higher counterpart recovery rate, $\mathrm{X}-\mathrm{O}$ fraction, and lower false-match probability (see Table 1 ) at the current depth. Deep HST WFC3/IR surveys or the forthcoming James Webb Space Telescope (JWST) will provide unprecedented NIR/IR data for source identification. Note that a deeper optical or NIR catalog will generally result in a larger false-match probability.

There are at least two possible approaches toward a smaller overall false-match probability: deeper X-ray observations and deeper radio observations. The probability distribution of angular separations, $f(r)$, is largely controlled by the X-ray positional errors (see Eq. 2) except when matching to the IR sources. Additional X-ray observations will improve the $\mathrm{X}$-ray positional accuracy and thus reduce the false-match probability. For example, as the $\approx 2 \mathrm{Ms} C D F-S$ is scheduled to be expanded to $\approx 4 \mathrm{Ms}$, the factor of $\approx 2$ increase in counts will reduce the areas of source positional error regions by $\approx 30 \%$ on average, reducing the number of spurious matches (at the current flux limit) by about this same factor. The VLA radio catalog has the highest $\mathrm{X}-\mathrm{O}$ fraction $(\approx 28 \%)$ and lowest false-match probability (see Table 1). However, it also has the lowest counterpart recovery rate $(\approx 20 \%)$. To examine if the high $\mathrm{X}-\mathrm{O}$ fraction of the radio catalog is intrinsic or rather just related to the low recovery rate (i.e., limited catalog depth), we compared it to the $\mathrm{X}-\mathrm{O}$ fractions of optical and NIR catalogs at similar effective depth. We limit the GOODS-S $z$-band catalog and MUSIC $K$-band catalog to brighter magnitudes $(z<21.0$ and $K<19.8)$ until the recovery rates of these two catalogs are the same as that of the radio catalog $(\approx 20 \%)$. The resulting X-O fractions are only $\approx 13 \%$ and $\approx 17 \%$ for these two catalogs, respectively. This suggests the X-ray sources are more strongly associated with the radio sources than optical or NIR sources at the current depth of the radio catalog, and thus deeper radio observations might well provide more reliable matches than the GOODS-S or MUSIC catalogs. The current radio catalog has a $5 \sigma$ limiting flux of $\approx 40 \mu \mathrm{Jy}$. Given the VLA $1.4 \mathrm{GHz}$ number counts estimated in Owen \& Morrison (2008), there will be $\approx 20$ times more radio sources at a flux limit of $5 \mu \mathrm{Jy}$. This radio source density (about 30 sources $\operatorname{arcmin}^{-2}$ ) is still $\approx 2-4$ times smaller than those of the optical and NIR catalogs, and we expect that such deeper radio catalogs can provide more reliable matches and thus reduce the overall false-match probability. The flux limit of $5 \mu \mathrm{Jy}$ can be easily achieved by facilities such as the Expanded Very Large Array (EVLA). The Atacama Large Millimeter Array (ALMA) at shorter wavelengths may also help with the goal of obtaining more reliable counterparts in a similar way.

\section{SUMMARY OF RESULTS}

1. We presented multiwavelength identifications of the 462 X-ray sources in the main Chandra source cata$\log$ for the $\approx 2$ Ms CDF-S. The optical-to-radio catalogs used for source matching include the WFI $R$-band, GOODS-S z-band, GEMS z-band, GOODS-S MUSIC $K$-band, MUSYC $K$-band, SIMPLE $3.6 \mu \mathrm{m}$, and VLA
1.4 GHz catalogs. The matching results are combined to create lists of primary and secondary counterparts. We identified reliable counterparts for 442 (95.7\%) of the X-ray sources, with an expected false-match probability of $\approx 6.2 \%$; these are the best identifications for sources at these X-ray fluxes. We also associated four additional likely counterparts given their small positional offsets to the relevant X-ray sources.

2. The likelihood-ratio method was used for source matching, which significantly reduces the false-match probability at faint magnitudes compared to the error-circle matching method. For example, the false-match probabilities at $R=26$ are $\approx 20 \%$ and $\approx 28 \%$ for the likelihood-ratio and error-circle methods, respectively. The resulting matches may differ by $\approx 20 \%$ for sources with $R>25.5$, where we expect to find some of the most interesting objects in the deep $\mathrm{X}$-ray surveys. The likelihood-ratio matching technique can also generate multiple counterpart candidates and calculate their relative reliability parameters, which are useful when considering multiwavelength identifications.

3. Among the optical-to-radio multiwavelength catalogs used for identification, the false-match probabilities range from $\approx 1-9 \%$, and the counterpart recovery rates range from $\approx 20-80 \%$. Given the current depths of the catalogs, the NIR catalogs (e.g., the MUSIC $K$-band catalog) can provide a large fraction of the counterparts for the X-ray sources with a relatively low false-match probability. Meanwhile, the radio catalog can provide the most secure counterparts with almost no spurious matches. Even after limiting the optical/NIR catalogs to similar effective depth as the radio catalog, the radio sources still have a significantly stronger association with the $\mathrm{X}$-ray sources (measured by the $\mathrm{X}-\mathrm{O}$ fraction parameter). Therefore, obtaining deeper radio observations will considerably benefit the matching reliability. Additional X-ray observations will reduce the $\mathrm{X}$-ray positional errors and thus also improve the identification reliability.

4. Of the 16 unidentified X-ray sources, eight are probably related to a star (one source), galaxy groups and clusters (four sources), off-nuclear sources (two sources), or any of a number of galaxies at the edge of the CDF-S field (one source). Another six sources detected at a WAVDETECT false-positive threshold of $1 \times 10^{-6}$ may be spurious $\mathrm{X}$-ray detections. The remaining two objects are likely genuine X-ray sources. Their X-ray-to-optical properties are comparable to the EXOs, though without any IR or NIR detections. They are likely to be AGNs at very high redshifts $(z>5)$, or one of them (source $345)$ could be an isolated neutron star.

5. We constructed a photometric catalog for the 446 identified X-ray sources including up to 42 bands of UV-to-IR data. We performed source deblending carefully for $\approx 10 \%$ of the sources in the IR bands and a few percent in the optical and NIR bands, obtaining the best-ever photometric data for these sources. High-quality photo-z's were then derived using the ZEBRA SED fitting code and a set of 265 galaxy, AGN, 
and galaxy/AGN hybrid templates. The ZEBRA training procedure corrects the SED templates to best represent the SEDs of real sources at different redshifts, which reduces template mismatches and improves the photo-z accuracy. Catalogs of photometric data and photo-z's are provided. We collected secure spec-z's for 220 of the X-ray sources. It appears that the majority of the X-ray sources without spec-z's are AGNs at relatively high redshifts $(z \approx 1-4)$.

6. The photo-z's are extremely accurate for sources with spec-z's. There are only 3 outliers out of 220 sources, and the photo-z's are accurate to within $\approx 1 \%\left(\sigma_{\mathrm{NMAD}}\right)$. We performed blind tests to derive a more realistic estimate of the overall photo-z accuracy for sources without spec-z's. We expect there are $\approx 9 \%$ outliers for the $\approx 110$ relatively brighter sources $(R \lesssim 26)$, and the outlier fraction will increase to $\approx 15-25 \%$ for the $\approx 110$ fainter sources $(R \gtrsim 26)$. The typical photo- $z$ accuracy is $\approx 6-7 \%\left(\sigma_{\mathrm{NMAD}}\right)$. The outlier fraction and photo-Z accuracy do not appear to have a redshift dependence (for $z \approx 0-4$ ). The photo-z quality is comparable to that of some recent photo-z's derived for AGNs at brighter $\mathrm{X}$-ray fluxes, and is significantly ( $\gtrsim 50 \%$ ) improved compared to that of the photo-Z's for the X-ray sources in the $\approx 1 \mathrm{Ms}$ CDF-S or $\approx 2 \mathrm{Ms}$ CDF-N.

We acknowledge financial support from CXC grant SP89003A/B (BL, WNB, YQX, MB, FEB, DR), NASA LTSA grant NAG5-13035 (WNB), the Royal Society and the Philip Leverhulme Fellowship Prize (DMA), and ASI-INAF grants I/023/05/00 and I/088/06 (AC). We thank R. Feldmann, R. Gilli, H. Hao, R.C. Hickox, K.I. Kellermann, N.A. Miller, and G. Pavlov for helpful discussions. We are grateful to A. Finoguenov, A. Grazian, N.A. Miller, and M. Nonino for kindly providing their catalogs.

\section{REFERENCES}

Aird, J., et al. 2010, MNRAS, 401, 2531

Alexander, D. M., et al. 2003, AJ, 126, 539

Arnaud, K. A. 1996, in ASP Conf. Ser., Vol. 101, Astronomical Data Analysis Software and Systems V, ed. G. H. Jacoby \& J. Barnes, 17

Balestra, I., et al. 2010, A\&A, in press (arXiv:1001.1115)

Barger, A. J., et al. 2003, AJ, 126, 632

Bauer, F. E., Alexander, D. M., Brandt, W. N., Schneider, D. P., Treister, E., Hornschemeier, A. E., \& Garmire, G. P. 2004, AJ, 128, 2048

Bertin, E., \& Arnouts, S. 1996, A\&AS, 117, 393

Brammer, G. B., van Dokkum, P. G., \& Coppi, P. 2008, ApJ, 686, 1503

Brandt, W. N., \& Hasinger, G. 2005, ARA\&A, 43, 827

Brusa, M., et al. 2005, A\&A, 432, 69

-. 2007, ApJS, 172, 353

-. 2009, A\&A, 507, 1277

Caldwell, J. A. R., et al. 2008, ApJS, 174, 136

Capak, P., et al. 2004, AJ, 127, 180

Cardamone, C. N., et al. 2008, ApJ, 680, 130

Ciliegi, P., Zamorani, G., Hasinger, G., Lehmann, I., Szokoly, G., \& Wilson, G. 2003, A\&A, 398, 901

Civano, F., et al. 2007, A\&A, 476, 1223

Comastri, A., et al. 2002, preprint (astro-ph/0203019)

Damen, M., et al. 2010, ApJ, submitted

Feldmann, R., et al. 2006, MNRAS, 372, 565

Fontanot, F., Cristiani, S., Monaco, P., Nonino, M., Vanzella, E., Brandt, W. N., Grazian, A., \& Mao, J. 2007, A\&A, 461, 39

Gawiser, E., et al. 2006, ApJ, 642, L13

Giacconi, R., et al. 2002, ApJS, 139, 369

Giavalisco, M., et al. 2004, ApJ, 600, L93

Gilli, R., et al. 2003, ApJ, 592, 721

Grazian, A., et al. 2006, A\&A, 449, 951

Hildebrandt, H., Wolf, C., \& Benítez, N. 2008, A\&A, 480, 703

Ilbert, O., et al. 2009, ApJ, 690, 1236

Kaplan, D. L. 2008, in AIP Conf. Ser., Vol. 983, 40 Years of Pulsars: Millisecond Pulsars, Magnetars and More, ed. C. Bassa, Z. Wang, A. Cumming, \& V. M. Kaspi, Am. Inst. Phys., New York, p. 331

Kellermann, K. I., Fomalont, E. B., Mainieri, V., Padovani, P., Rosati, P., Shaver, P., Tozzi, P., \& Miller, N. 2008, ApJS, 179, 71

Koekemoer, A. M., et al. 2004, ApJ, 600, L123

Komatsu, E., et al. 2009, ApJS, 180, 330

Laird, E. S., et al. 2009, ApJS, 180, 102

Le Fèvre, O., et al. 2004, A\&A, 428, 1043

Lehmer, B. D., Brandt, W. N., Hornschemeier, A. E., Alexander, D. M., Bauer, F. E., Koekemoer, A. M., Schneider, D. P., \& Steffen, A. T. 2006, AJ, 131, 2394

Lehmer, B. D., et al. 2005, ApJS, 161, 21

Luo, B., et al. 2008, ApJS, 179, 19 (L08)

Mainieri, V., et al. 2005, A\&A, 437, 805
-. 2008, ApJS, 179, 95

Maronna, R. A., Martin, R. D., \& Yohai, V. J. 2006, Robust Statistics: Theory and Methods (1st ed.; UK: John Wiley \& Sons)

Matt, G. 2002, Philos. Trans. R. Soc. London Ser. A, 360, 2045

Mignoli, M., et al. 2005, A\&A, 437, 883

Miller, N. A., Fomalont, E. B., Kellermann, K. I., Mainieri, V., Norman, C., Padovani, P., Rosati, P., \& Tozzi, P. 2008, ApJS, 179, 114

Moran, E. C., Filippenko, A. V., \& Chornock, R. 2002, ApJ, 579, L71

Morrissey, P., et al. 2005, ApJ, 619, L7

Nonino, M., et al. 2009, ApJS, 183, 244

Oke, J. B., \& Gunn, J. E. 1983, ApJ, 266, 713

Owen, F. N., \& Morrison, G. E. 2008, AJ, 136, 1889

Polletta, M., et al. 2007, ApJ, 663, 81

Popesso, P., et al. 2009, A\&A, 494, 443

Popov, S. B., Colpi, M., Prokhorov, M. E., Treves, A., \& Turolla, R. 2000, ApJ, 544, L53

Popov, S. B., Pons, J. A., Miralles, J. A., Boldin, P. A., \& Posselt, B. 2010, MNRAS, 401, 2675

Ravikumar, C. D., et al. 2007, A\&A, 465, 1099

Richards, G. T., et al. 2006, ApJS, 166, 470

Rigby, J. R., Rieke, G. H., Donley, J. L., Alonso-Herrero, A., \& PérezGonzález, P. G. 2006, ApJ, 645, 115

Rodighiero, G., Cimatti, A., Franceschini, A., Brusa, M., Fritz, J., \& Bolzonella, M. 2007, A\&A, 470, 21

Salvato, M., et al. 2009, ApJ, 690, 1250

Severgnini, P., et al. 2003, A\&A, 406, 483

Silverman, J. D., et al. 2008, ApJ, 675, 1025

Sutherland, W., \& Saunders, W. 1992, MNRAS, 259, 413

Szokoly, G. P., et al. 2004, ApJS, 155, 271

Taylor, E. N., et al. 2009, ApJS, 183, 295

Tozzi, P., et al. 2006, A\&A, 451, 457

Trouille, L., Barger, A. J., Cowie, L. L., Yang, Y., \& Mushotzky, R. F. 2009, ApJ, 703, 2160

Trump, J. R., et al. 2009, ApJ, 706, 797

Vanzella, E., et al. 2006, A\&A, 454, 423

-. 2008, A\&A, 478, 83

Vignali, C., Bauer, F. E., Alexander, D. M., Brandt, W. N., Hornschemeier, A. E., Schneider, D. P., \& Garmire, G. P. 2002, ApJ, 580, L105

Waddington, I., Windhorst, R. A., Cohen, S. H., Partridge, R. B., Spinrad, H., \& Stern, D. 1999, ApJ, 526, L77

Wolf, C., Hildebrandt, H., Taylor, E. N., \& Meisenheimer, K. 2008, A\&A, 492, 933

Wolf, C., et al. 2004, A\&A, 421, 913

Yuan, F., \& Narayan, R. 2004, ApJ, 612, 724

Zheng, W., et al. 2004, ApJS, 155, 73 
TABLE 1

Summary of the Likelihood-Ratio Matching Parameters AND Results

\begin{tabular}{|c|c|c|c|c|c|c|c|c|c|c|c|c|c|c|c|c|c|}
\hline $\begin{array}{l}\text { Catalog } \\
\text { (1) }\end{array}$ & $\begin{array}{l}\text { Det. Thresh } \\
\text { (2) }\end{array}$ & $\begin{array}{l}\text { Depth } \\
\text { (3) }\end{array}$ & $\begin{array}{l}\text { Solid Angle } \\
\text { (4) }\end{array}$ & $\begin{array}{l}N_{\mathrm{o}} \\
(5)\end{array}$ & $\begin{array}{l}\sigma_{\mathrm{o}} \\
(6)\end{array}$ & $\begin{array}{l}L_{\text {th }} \\
(7)\end{array}$ & $\begin{array}{c}R \\
(8)\end{array}$ & $\begin{array}{l}C \\
(9)\end{array}$ & $\begin{array}{l}N_{\mathrm{X}} \\
(10)\end{array}$ & $\begin{array}{l}N_{\mathrm{ID}} \\
(11)\end{array}$ & $\begin{array}{l}N_{\text {NoID }} \\
(12)\end{array}$ & $\begin{array}{l}N_{\text {Multi }} \\
\text { (13) }\end{array}$ & $\begin{array}{l}N_{\text {False }} \\
(14)\end{array}$ & $\begin{array}{l}\text { False \% } \\
\text { (15) }\end{array}$ & $\begin{array}{c}\text { Recovery \% } \\
\text { (16) }\end{array}$ & $\begin{array}{l}\mathrm{X}-\mathrm{O} \% \\
(17)\end{array}$ & $\begin{array}{l}N_{\text {Pri }} \\
(18)\end{array}$ \\
\hline WFI $R$ & $2 \sigma$ & 27.3 & 1420 & 30345 & $0.1^{\prime \prime}$ & 0.55 & 0.97 & 0.73 & 462 & 344 & 118 & 2 & 32 & $9 \%$ & $67.5 \%$ & $1.0 \%$ & 19 \\
\hline GOODS-S $z$ & $0.6 \sigma$ & 28.2 & 160 & 33955 & $0.1^{\prime \prime}$ & 0.80 & 0.93 & 0.82 & 311 & 259 & 52 & 8 & 23 & $9 \%$ & $75.9 \%$ & $0.7 \%$ & 220 \\
\hline GEMS $z$ & $1.7 \sigma$ & 27.3 & 830 & 22016 & $0.1^{\prime \prime}$ & 2.35 & 0.97 & 0.67 & 462 & 312 & 150 & 5 & 14 & $4 \%$ & $64.5 \%$ & $1.4 \%$ & 89 \\
\hline MUSIC $K$ & $1 \sigma$ & 23.8 & 140 & 13595 & $0.1^{\prime \prime}$ & 1.30 & 0.93 & 0.84 & 262 & 223 & 39 & 4 & 8 & $4 \%$ & $82.1 \%$ & $1.6 \%$ & 14 \\
\hline MUSYC $K$ & 23.5/arcsec ${ }^{2}$ & 22.4 & 970 & 6998 & $0.2^{\prime \prime}$ & 0.85 & 0.99 & 0.70 & 462 & 326 & 136 & 0 & 12 & $4 \%$ & $68.0 \%$ & $4.5 \%$ & 9 \\
\hline SIMPLE $3.6 \mu \mathrm{m}$ & $2 \sigma$ & 23.8 & 1640 & 22095 & $0.3^{\prime \prime}$ & 0.20 & 0.99 & 0.89 & 462 & 414 & 48 & 0 & 32 & $8 \%$ & $82.7 \%$ & $1.7 \%$ & 12 \\
\hline VLA $1.4 \mathrm{GHz}$ & $5 \sigma$ & 19.9 & 1170 & 338 & $0.1^{\prime \prime}$ & 2.25 & 0.99 & 0.20 & 462 & 94 & 368 & 0 & 0.5 & $1 \%$ & $20.2 \%$ & $27.7 \%$ & 83 \\
\hline
\end{tabular}

NOTE. - Col. (1): ONIR catalog. References. - WFI and GOODS-S: Giavalisco et al. (2004); GEMS:[Caldwell et al. (2008); MUSIC: Grazian et al. (2006); MUSYC: Taylor et al. (2009); SIMPLE: Damen et al. (2010); VLA: Miller et al. (2008). Col. (2): The minimum threshold used for source detection in the catalog. Note that in some cases multiple searches (3): Catalog depth in AB flux densities, $m(\mathrm{AB})=-2.5 \log \left(f_{\nu}\right)-48.60$. Col. (4): Catalog solid-angle coverage in units of $\operatorname{arcmin}^{2}$. The GEMS catalog includes GOODS-S v1 data. All the catalogs cover the entire $\approx 2 \mathrm{Ms} \mathrm{CDF-S}\left(\approx 436 \operatorname{arcmin}^{2}\right)$ except for the GOODS-S and MUSIC surveys. Col. (5): Number of ONIR sources in the $\approx 2 \mathrm{Ms}$ CDF-S region. Col. (6): $1 \sigma$ positional error of the ONIR sources. Col. (7): Threshold value for the likelihood ratio to discriminate between spurious and real identifications. The threshold value is catalog dependent, and generally scales with the typical values of likelihood ratios (see Eq. 1), which usually increase when the catalog depth or positional errors decrease. Col. (8): Sample reliability. See $\$ 2.2$ for details. Col. (9): Sample completeness. See $\$ 2.2$ for details. Col. (10): Total number of X-ray sources that are within the coverage of the ONIR catalog. Col. (11): Number of X-ray sources identified with at least one ONIR counterpart in this catalog. Col. (12): Number of X-ray sources not identified, which equals Col. (10) minus Col. (11). Col. (13): Number of X-ray sources identified with two ONIR counterparts in this catalog. Col. (14): Expected number of false matches. See $\$ 2.3$ for details. Col. (15): False-match probability. See $\$ \sqrt{2.3}$ for details. Col. (16): Counterpart recovery rate, defined as the expected number of true counterparts $\left(N_{\mathrm{ID}}-N_{\mathrm{F}}\right)$ divided by the number of X-ray sources ( $\left.N_{\mathrm{X}}\right)$. Col. (17): The fraction of ONIR objects that are detected in the X-ray, defied as the expected

(18): Number of primary counterparts selected from this catalog. See $\$ 2.2$ for details. 
TABLE 2

LIST OF THE PRIMARY COUNTERPARTS

\begin{tabular}{ccccccccccccc}
\hline \hline ID & $\begin{array}{c}\sigma_{\mathrm{X}} \\
(1)\end{array}$ & $\begin{array}{c}\text { ONIR ID } \\
(2)\end{array}$ & $\begin{array}{c}\text { ONIR RA } \\
(4)\end{array}$ & $\begin{array}{c}\text { ONIR Dec } \\
(5)\end{array}$ & $\begin{array}{c}\sigma_{\mathrm{o}} \\
(6)\end{array}$ & $\begin{array}{c}\text { Dis } \\
(7)\end{array}$ & $\begin{array}{c}\text { Dis/ } \sigma \\
(8)\end{array}$ & $\begin{array}{c}R_{\mathrm{c}} \\
(9)\end{array}$ & $\begin{array}{c}\text { CAT } \\
(10)\end{array}$ & $\begin{array}{c}\text { MAG } \\
(11)\end{array}$ & $\begin{array}{c}\text { Multi } \\
(12)\end{array}$ & $\begin{array}{c}\text { Note } \\
(13)\end{array}$ \\
\hline 1 & 0.77 & 24484 & 52.8924 & -27.8347 & 0.1 & 0.89 & 1.14 & 0.88 & GEMS & 24.14 & 0 & $\ldots$ \\
2 & 0.94 & 21150 & 52.8990 & -27.8597 & 0.1 & 0.29 & 0.31 & 0.97 & GEMS & 22.27 & 0 & $\ldots$ \\
3 & 0.65 & 24081 & 52.9171 & -27.7962 & 0.1 & 0.44 & 0.68 & 0.98 & GEMS & 22.26 & 0 & $\ldots$ \\
4 & 0.55 & 0 & 0 & 0 & 0 & 0 & 0 & 0 & $\cdots$ & 0 & 0 & Edge
\end{tabular}

NOTE. - Table 2 is presented in its entirety in the electronic edition. An abbreviated version of the table is shown here for guidance as to its form and content. The full table contains 13 columns of information on primary counterparts to the $462 \mathrm{X}$-ray sources.

TABLE 3

LIST OF THE SECONDARY COUNTERPARTS

\begin{tabular}{ccccccccccc}
\hline \hline ID & $\begin{array}{c}\sigma_{\mathrm{X}} \\
(1)\end{array}$ & $\begin{array}{c}\text { ONIR ID } \\
(2)\end{array}$ & $\begin{array}{c}\text { ONIR RA } \\
(4)\end{array}$ & $\begin{array}{c}\text { ONIR Dec } \\
(5)\end{array}$ & $\begin{array}{c}\sigma_{\mathrm{o}} \\
(6)\end{array}$ & $\begin{array}{c}\text { Dis } \\
(7)\end{array}$ & $\begin{array}{c}\text { Dis/ } \sigma \\
(8)\end{array}$ & $\begin{array}{c}R_{\mathrm{c}} \\
(9)\end{array}$ & $\begin{array}{c}\text { CAT } \\
(10)\end{array}$ & $\begin{array}{c}\text { MAG } \\
(11)\end{array}$ \\
\hline 21 & 0.51 & 24151 & 52.9602 & -27.8768 & 0.3 & 1.38 & 2.34 & 0.95 & SIMPLE & 21.40 \\
25 & 0.39 & 35263 & 52.9648 & -27.7648 & 0.3 & 1.56 & 3.16 & 0.65 & SIMPLE & 21.99 \\
31 & 0.62 & 23319 & 52.9752 & -27.8349 & 0.1 & 0.73 & 1.16 & 0.63 & GEMS & 21.23 \\
41 & 0.43 & 30803 & 52.9906 & -27.7022 & 0.1 & 0.34 & 0.78 & 0.98 & GEMS & 23.39
\end{tabular}

Note. - Table 3 is presented in its entirety in the electronic edition. An abbreviated version of the table is shown here for guidance as to its form and content. The full table contains 11 columns of information on the secondary counterparts of 72 X-ray sources $(77$ lines). A high $R_{\mathrm{c}}$ value for the secondary counterpart indicates that the primary and secondary counterparts were identified in different catalogs.

TABLE 4

PHOTOMETRIC CATALOG

\begin{tabular}{lccccccccc}
\hline \hline ID & GALEX & & & & \multicolumn{5}{c}{ VIMOS } \\
\multicolumn{1}{c}{ NUV } & $\begin{array}{c}\text { Err } \\
(1)\end{array}$ & $\begin{array}{c}\text { Flag } \\
(2)\end{array}$ & $\begin{array}{c}\text { FUV } \\
(5)\end{array}$ & $\begin{array}{c}\text { Err } \\
(6)\end{array}$ & $\begin{array}{c}\text { Flag } \\
(7)\end{array}$ & $\begin{array}{c}U \\
(8)\end{array}$ & $\begin{array}{c}\text { Err } \\
(9)\end{array}$ & $\begin{array}{c}\text { Flag } \\
(10)\end{array}$ \\
\hline Median & 23.39 & 1.32 & 99 & 23.87 & 1.66 & 62 & 25.81 & 3.79 & 440 \\
1 & -26.89 & 99.00 & 1 & -27.25 & 99.00 & 1 & -28.53 & 99.00 & 1 \\
2 & 23.36 & 0.08 & 1 & -27.24 & 99.00 & 1 & 99.00 & 99.00 & 0 \\
3 & -26.90 & 99.00 & 1 & -27.23 & 99.00 & 1 & 22.32 & 0.01 & 0 \\
4 & 99.00 & 99.00 & 0 & 99.00 & 99.00 & 0 & 99.00 & 99.00 & 0 \\
\hline
\end{tabular}

Note. - Table 4 is presented in its entirety in the electronic edition. An abbreviated version of the table is shown here for guidance as to its form and content. The full table contains 181 columns of photometric data for the 462 X-ray sources (Rows 2-463, including 16 sources with no counterparts). The first row includes the median magnitude information for each band. All the photometric data are given in units of $\mathrm{AB}$ magnitudes, and are set to "99" if not available; upper limits are indicated by the "-" sign.

TABLE 5

Photometric Redshift CATALOG

\begin{tabular}{cccccccccccc}
\hline \hline ID & $\begin{array}{c}\text { Spec-z } \\
(1)\end{array}$ & $\begin{array}{c}\text { Photo-z } \\
(2)\end{array}$ & $\begin{array}{c}\text { zLower } \\
(3)\end{array}$ & $\begin{array}{c}\text { zUpper } \\
(4)\end{array}$ & $\begin{array}{c}\text { \#Detect } \\
(5)\end{array}$ & $\begin{array}{c}\text { \#Filter } \\
(7)\end{array}$ & $\begin{array}{c}\chi_{\text {dof }}^{2} \\
(8)\end{array}$ & $\begin{array}{c}\text { Photo-zalt } \\
(9)\end{array}$ & $\begin{array}{c}\chi_{\text {dof,alt }}^{2} \\
(10)\end{array}$ & $\begin{array}{c}\text { Op. Det. } \\
(11)\end{array}$ & $\begin{array}{c}\text { zRef } \\
(12)\end{array}$ \\
\hline 1 & -1.000 & 1.102 & 1.045 & 1.114 & 13 & 16 & 1.8 & -1.0 & -1.0 & 1 & -1 \\
2 & -1.000 & 1.626 & 1.620 & 1.633 & 26 & 27 & 23.6 & -1.0 & -1.0 & 1 & -1 \\
3 & 2.709 & 2.714 & 2.712 & 2.715 & 26 & 28 & 8.3 & -1.0 & -1.0 & 1 & 8 \\
4 & -1.000 & -1.000 & -1.000 & -1.000 & 0 & 0 & -1.0 & -1.0 & -1.0 & 0 & -1
\end{tabular}

Note. - Table 5 is presented in its entirety in the electronic edition. An abbreviated version of the table is shown here for guidance as to its form and content. The full table contains 12 columns of information for the $462 \mathrm{X}$-ray sources (including 16 sources with no counterparts and six stars). 